\title{
NESTED AND SINGLE BARS IN SEYFERT AND NON-SEYFERT GALAXIES
}

\author{
SEPPO LAINE ${ }^{1}$ \\ Department of Physics and Astronomy, University of Kentucky, Lexington, KY 40506-0055; laine@stsci.edu \\ ISAAC SHLOSMAN ${ }^{2,3}$ \\ JILA, University of Colorado, Box 440, Boulder, CO 80309-0440; shlosman@pa.uky.edu \\ JOHAN H. KNAPEN \\ Isaac Newton Group of Telescopes, Apartado 321, E-38700 Santa Cruz de La Palma, Spain; and Department of Physical Sciences, \\ University of Hertfordshire, Hatfield, Hertfordshire AL10 9AB, UK; knapen@ing.iac.es \\ AND
}

REYNIER F. PELETIER

School of Physics and Astronomy, University of Nottingham, Nottingham NG7 2RD, UK; Reynier.Peletier@nottingham.ac.uk Received 2001 June 20; accepted 2001 October 11

\begin{abstract}
We analyze the observed properties of nested and single stellar bar systems in disk galaxies. The 112 galaxies in our sample comprise the largest matched Seyfert versus non-Seyfert galaxy sample of nearby galaxies with complete near-infrared or optical imaging sensitive to length scales ranging from tens of parsecs to tens of kiloparsecs. The presence of bars is deduced by fitting ellipses to isophotes in Hubble Space Telescope (HST) H-band images up to $10^{\prime \prime}$ radius and in ground-based near-infrared and optical images outside the $H$-band images. This is a conservative approach that is likely to result in an underestimate of the true bar fraction. We find that a significant fraction of the sample galaxies, $17 \% \pm 4 \%$, have more than one bar, and that $28 \% \pm 5 \%$ of barred galaxies have nested bars. The bar fractions appear to be stable according to reasonable changes in our adopted bar criteria. For the nested bars, we detect a clear division in length between the large-scale (primary) bars and small-scale (secondary) bars, in both absolute and normalized (to the size of the galaxy) length. We argue that this bimodal distribution can be understood within the framework of disk resonances, specifically the inner Lindblad resonances (ILRs), which are located where the gravitational potential of the innermost galaxy switches effectively from three-dimensional to two-dimensional. This conclusion is further strengthened by the observed distribution of the sizes of nuclear rings which are dynamically associated with the ILRs. While primary bar sizes are found to correlate with the host galaxy sizes, no such correlation is observed for the secondary bars. Moreover, we find that secondary bars differ morphologically from single bars. Our matched Seyfert and non-Seyfert samples show a statistically significant excess of bars among the Seyfert galaxies at practically all length scales. We confirm our previous results that bars are more abundant in Seyfert hosts than in non-Seyfert galaxies and that Seyfert galaxies always show a preponderance of "thick" bars compared to the bars in non-Seyfert galaxies. Finally, no correlation is observed between the presence of a bar and that of companion galaxies, even relatively bright ones. Overall, since star formation and dust extinction can be significant even in the $H$ band, the stellar dynamics of the central kiloparsec cannot always be revealed reliably by the use of near-infrared surface photometry alone.

Subject headings: galaxies: evolution — galaxies: nuclei — galaxies: Seyfert — galaxies: spiral galaxies: statistics - infrared: galaxies

On-line material: machine-readable tables
\end{abstract}

\section{INTRODUCTION}

While a substantial effort has been spent on understanding the prevalence and properties of kiloparsec-scale bars, little is known about bars on subkiloparsec scales. These "inner" or "nuclear" bars were first discovered as optical isophote twists in the central regions of barred galaxies (e.g., de Vaucouleurs 1974; Sandage \& Brucato 1979; Kormendy 1982) and interpreted as triaxial bulges of barred galaxies. Later ground-based studies at higher resolution have revealed more galaxies with nuclear bars, lying inside large galactic bars (e.g., Buta \& Crocker 1991, 1993; Shaw et al. 1993, 1995; Knapen et al. 1995b; Wozniak et al. 1995;

\footnotetext{
${ }^{1}$ Present address: Space Telescope Science Institute, 3700 San Martin Drive, Baltimore, MD 21218.

2 JILA Visiting Fellow.

${ }^{3}$ Permanent address: Department of Physics and Astronomy, University of Kentucky, Lexington, KY 40506-0055.
}

Möllenhoff, Matthias, \& Gerhard 1995; Friedli et al. 1996; Jungwiert, Combes, \& Axon 1997; Mulchaey \& Regan 1997; Elmegreen, Chromey, \& Santos 1998; Jogee, Kenney, \& Smith 1998, 1999; Knapen, Shlosman, \& Peletier 2000, hereafter KSP00; Márquez et al. 2000; Greusard et al. 2000). Some of the earlier studies have been summarized by Buta \& Combes (1996).

Although the first detections of subkiloparsec bars were made in stellar light, these objects can contain arbitrary fractions of gas and in extreme cases can be dynamically dominated by molecular gas, as evident in their detection in interferometric $2.6 \mathrm{~mm} \mathrm{CO}$ emission and in the nearinfrared (NIR) lines of $\mathrm{H}_{2}$ emission (e.g., Ishizuki et al. 1990; Devereux, Kenney, \& Young 1992; Forbes, Kotilainen, \& Moorwood 1994; Mirabel et al. 1999; Kotilainen et al. 2000; Maiolino et al. 2000). CO observations have a rather low spatial resolution (at best just below 1" FWHM) but do allow the determination of the offset angle between the 
large-scale stellar and the small-scale gaseous bar. It is not yet clear whether stellar-dominated and gas-dominated subkiloparsec bars have a common origin or describe a concurrent phenomenon. In this paper we focus on stellar bars, analyzing their properties based on NIR and optical starlight.

The high spatial resolution capability provided by the Hubble Space Telescope (HST) has enabled studies of galaxy centers with about 0 ". 1 resolution. More embedded nuclear bars have been detected in these observations (e.g., van den Bosch \& Emsellem 1998; Erwin \& Sparke 1999a, 1999b; Regan \& Mulchaey 1999; Martini \& Pogge 1999; Colina \& Wada 2000; Emsellem \& Ferruit 2000) using various techniques, but most of these papers only discuss one galaxy, apart from those by Regan \& Mulchaey (1999) and Martini \& Pogge (1999), where 12 and 24 galaxies were considered, respectively.

Because the existence of a nested bar system is intrinsically a time-dependent phenomenon, no single-periodic orbits, which form the "backbone" of a barred galaxy, can dominate the stellar dynamics. Maciejewski \& Sparke (2000) discuss a special class of double-bar quasi-periodic orbits, but the exact fraction of the total phase space occupied by these orbits is unknown, and whether they are significantly populated is unclear. Most of these orbits can only host stars: because nearly all of them intersect, they are not suitable for the gas and cannot support the steady state gas motions. Because of these and additional reasons, offset dust lanes, which characterize the gas motions in the large stellar bars, do not form in nuclear bars and cannot be used as indicators of bar presence (Shlosman \& Heller 2002). The sparseness of nuclear bars in the recent NIR snapshot survey by Regan \& Mulchaey (1999) and Martini \& Pogge (1999), who attempted to find subkiloparsec bars based on the offset dust lanes, should therefore not be surprising, as these studies postulated identical gas flows in large- and small-scale bars.

This work is the first attempt to determine the statistics of nested bars in disk galaxies and to compare nested bars with single bars in Seyfert and non-Seyfert host galaxies. For this we have taken a large sample of nearby spiral galaxies with available $H S T$ archive NICMOS images in the $H$ band, added ground-based data of the outer disks, and analyzed all the images for the presence of bars using fits of ellipses to isophotes. This is the largest high spatial resolution sample analyzed so far. As a by-product, we are able to test our previous results (KSP00) on the large-scale bar fractions in Seyfert and non-Seyfert galaxies, with improved statistical significance.

We give the definitions of the bar concepts that we use throughout our paper in $\S 2$ and describe our samples and the analysis of the images in $\S 3$. Results on nested and single bars are presented in $\S 4$, and the differences between Seyfert and non-Seyfert bar properties are given in $\S 5$. Discussion and conclusions are provided in $\S \S 6$ and 7, respectively.

\section{DEFINING ASPECTS OF NUCLEAR BARS}

We define nested bar systems as those with more than one bar, stellar or gaseous, and focus here on purely stellar bars. Although the terms "nuclear bar" or "inner bar" have been used in the literature to refer to bars other than the largest bar in a system, these terms are loosely defined. Overall they refer to bars lying in scarcely resolved nuclear regions of disk galaxies, generally within $\sim 1 \mathrm{kpc}$. Naturally the names do not reflect any special physical properties of these bars. To avoid further ambiguities with bar definitions, we use the following notation.

We first distinguish between large- and small-scale bars in double-barred systems. The former are referred to as "primary," while the latter are called "secondary." The theoretical rationale behind these definitions is that secondary bars are believed to form as a result of radial gas inflow due to the large-scale bar and, therefore, are expected to be confined within the inner Lindblad resonances (ILRs), which naturally limit their size to $\sim 1 \mathrm{kpc}$ (Shlosman, Frank, \& Begelman 1989). Within this framework, molecular gas accumulation in the vicinity of the ILRs is susceptible to a global gravitational (bar) instability which will affect stars as well, by dragging them along. Secondary bars would then form with a pattern speed which is higher than that of the primary bar. This view was supported by Pfenniger \& Norman (1990), who, using weakly dissipative equations of motion for a test particle, analyzed the properties of double-barred galaxies. In particular, they argued that the corotation radius of the secondary bar should coincide with the ILR of the primary bar in order to reduce the fraction of chaotic orbits in the resonance neighborhood. This suggestion was made on purely theoretical grounds. It means that the ILRs serve as a dynamical separator between primary and secondary bars, a point we address observationally in this paper. Finally, bars in galaxies which host only one bar are called "single bars," independent of their physical length.

\section{SAMPLE AND DATA ANALYSIS}

\subsection{Sample}

Our Seyfert sample consists of most of the Seyfert galaxies in the local universe $\left(v_{\text {hel }}<6000 \mathrm{~km} \mathrm{~s}^{-1}\right)$ that have been observed in the F160W $(H)$ band with HST. Most of the Seyfert galaxies come from the relatively large samples of Mulchaey (part of which was published by Regan \& Mulchaey 1999), Stiavelli (Seigar et al. 2000), Pogge (Martini \& Pogge 1999), and Peletier (Peletier et al. 1999a). A few well-known Seyfert galaxies were added to our Seyfert sample: NGC 1068 (Thompson \& Corbin 1999), NGC 3227 (Quillen et al. 1999), NGC 4151 (R. Thompson 2001, unpublished), NGC 5548 (Quillen et al. 2001), and NGC 7469 (Scoville et al. 2000). As in KSP00, we removed highly inclined galaxies by requiring that the apparent axial ratio, as obtained from the RC3 (de Vaucouleurs et al. 1991), had to be greater than 0.45 , since the detection of nonaxisymmetric structures in highly inclined galaxies is problematic. We included galaxies which have been classified in RC3 with Hubble types S0-Sc because there are very few Seyfert galaxies of later types. Since we are interested in the central kiloparsec of disk galaxies, we did not discard interacting galaxies, except when the interaction was accompanied by a strong morphological distortion. Note that the effect of interactions on the galactic morphology in the central kiloparsec generally is not significant (unless it is a full-fledged merger) because of short relaxation timescales in the circumnuclear region. We further discuss the influence of companion galaxies in $\S 6$. Galaxies which had been so badly centered that their nucleus was lying close to the edge of the NICMOS field were excluded. This left us with a final sample of 56 Seyfert 
galaxies. We then matched the Seyfert sample by a control sample of 56 non-Seyfert galaxies, also observed in the F160W band of NICMOS.

\subsection{Sample Matching}

We constructed the control sample to have a similar distribution to the Seyfert sample in the following four parameters: absolute $B$ magnitude, distance, axis ratio, and morphological type. Our control galaxies were selected from a sample of 95 non-Seyfert galaxies for which HST $H$-band images existed. To match the distributions of the non-Seyfert galaxies to those of the Seyfert galaxies in the aforementioned four parameters, we divided the Seyfert galaxies into two magnitude bins, two distance bins, five axis ratio bins, and six morphological type bins. We then eliminated non-Seyfert galaxies in bins that had an excess of them compared to the Seyfert galaxies. This procedure was repeated until a match was found that produced the smallest total difference in the bin distributions between the Seyfert and non-Seyfert galaxies. In the absolute $B$ magnitude, which had been derived from the apparent $B_{T, 0}$ magnitude in RC3 and the distance, we split the samples at magnitude -20.4 , which divides the samples into two bins with roughly equal numbers of galaxies. Changes in this dividing magnitude of more than 0.1 would produce a very different (and unequal) division of the galaxies in the two magnitude bins. The distance was obtained from Tully's (1988) Nearby Galaxies Catalog or from the heliocentric velocity using the Hubble law and a Hubble constant of 75 $\mathrm{km} \mathrm{s}^{-1} \mathrm{Mpc}^{-1}$. We divided the samples into two distance bins with the bin separation at $28 \mathrm{Mpc}$. Changing the dividing boundary by more than $2 \mathrm{Mpc}$ would produce very different (and uneven) partitions in the two bins. For the axis ratio, the values separating the bins are $0.6,0.7,0.8$, and 0.9 . For the morphological type, we used bins in the numerical Hubble Type T given in RC3. Graphical representations and tables illustrating our sample matching are given in Appendix A.

\subsection{Data Reduction}

We chose to analyze $H$-band images only. $K$-band images would likely be more reliable since they suffer less from the disturbing effects of dust extinction, but unfortunately $K$-band $H S T$ images were not available for the majority of our sample galaxies. We started with the data produced by the NICMOS pipeline, as available in the HST archive. Further reduction involved the masking of artifacts from the NICMOS images, and sometimes the removal of an additional pedestal level, to make the background flat. The artifacts often included the coronagraphic hole of the NICMOS camera and involved masking of the central columns of the images which were mosaicked by the NICMOS calibration pipeline. Nearby stars were also masked out. In case of bright point-source nuclei, we generated a point-spread function (PSF) with the Tiny Tim software and ran a few iterations of Lucy deconvolution. In extreme cases of diffraction, we used the IRAF task CPLUCY to perform the deconvolution in the central region. This deconvolution eliminated the original diffraction rings completely. The outer spikes of the PSF were manually masked out.

\subsection{Bar Detection and Classification}

Since we aim to detect all the bars in the sample, whether they are secondary, primary, or single bars, the $H S T$ data have been extended to larger radii. Ideally, we preferred to have NIR images with a large field of view, thus covering the galaxies completely. Since such images, unfortunately, were not available, we applied a different solution. Just beyond the range of the NICMOS images, we use data from the 2MASS all-sky survey. Although it is not a very deep survey and has a relatively low spatial resolution (about $2^{\prime \prime}$ ), its $H$ band is compatible, and it allows us to cover the radial range between typically $5^{\prime \prime}$ and $30^{\prime \prime}$. Farther out we use optical images, either CCD images from various data archives or Digitized Sky Survey (DSS) images. Although optical images are affected considerably more by dust extinction and star formation, thus making bar classification much more difficult, most of the dust is probably found in the inner regions (e.g., Giovanelli et al. 1994; Peletier et al. 1995), and not many bars will be missed.

We used the GALPHOT package (see Jørgensen, Franx, \& Kjaergaard 1992), run under the IRAF ${ }^{4}$ environment, to fit elliptical isophotes to the galaxy images. We also followed closely the procedure given in Peletier et al. (1999b). The center of the isophotes was given an initial guess based on the location of the peak in the image, but the fitting program had the freedom to move the center of the fitted ellipses. We fitted ellipses usually in multiplicative (1.1) radial increments. The fitted position angle in the images was transformed to true position angle (measured east of north on the sky) by using the header information in the HST images. A few of our sample galaxies were not well centered in the field of view of the camera, and therefore it was not possible to reliably fit these galaxies to the edge of the field of view because only a small fraction of the ellipses would lie on the image (NICMOS2, which was used for most of the images, has a field of view of $19^{\prime \prime} .2 \times 19{ }^{\prime \prime} .2$ ). Similarly, ellipses were fitted to the images from other sources (2MASS, DSS, etc.). The fitted ellipses were then deprojected using a two-dimensional deprojection. For this we assumed that the outer parts of the galaxies are flat circular disks, with the inclination given by the axis ratio in NED.

Next, we describe our method of bar detection on all possible length scales in the images. To classify a galaxy as barred, we use the criteria set in KSP00, namely, a bar is revealed by a significant rise in ellipticity $(1-b / a)$, followed by a significant fall, while the position angle of the major axis of the fitted ellipse is roughly constant. To quantify this a little further, we require that the ellipticity variation has an amplitude of at least 0.1 (increase and decrease) while the position angle varies by less than $20^{\circ}$. To be conservative, we did not follow the second criterion of KSP00, namely, position angle twists of more than $75^{\circ}$ accompanied by ellipticities above the 0.1 level. We have checked that the bar fractions are stable against reasonable changes in the bar criteria, such as a change of $20 \%$ in ellipticity amplitude requirement and a change of $50 \%$ in the constancy of the position angle requirement. The bar length was defined to be the radius where the fitted bar ellipticity peaks. The bar ellipticity was defined as the maximum ellipticity of a detected bar. Tabulated properties of the bars and graphical

\footnotetext{
${ }^{4}$ IRAF is distributed by the National Optical Astronomy Observatory, which is operated by the Association of Universities for Research in Astronomy, Inc., under cooperative agreement with the National Science Foundation.
} 
representations of the deprojected ellipse fits are given in Appendix B.

\section{OBSERVED NESTED AND SINGLE BAR PROPERTIES}

\subsection{Nested Bars}

\subsubsection{Overall Statistics}

In total we find that 69 of our 112 galaxies have at least one bar $(62 \% \pm 5 \%)$. We use Poisson statistics to give an uncertainty estimate of our numbers, estimated from the formula $\sigma=[f(1-f / N)]^{1 / 2}$, where $f$ is the quantity that is measured and $N$ is the sample size in which this quantity is searched $(f=69$ and $N=112$ above). There are 12 $(21 \% \pm 5 \%)$ Seyfert and seven $(13 \% \pm 4 \%)$ non-Seyfert galaxies in our samples which have nested bar systems. These include two triple-barred systems among the Seyfert galaxies. In triple-barred systems we classify the outermost bar as a primary bar and the two innermost bars as secondaries. Altogether we have found secondary or primary bars in 19 $(17 \% \pm 4 \%)$ galaxies. This represents the bar fraction in spiral galaxies which have a morphological type distribution of the Seyfert hosts in our sample, making our results somewhat biased toward early-type spirals.

\subsubsection{Bar Size Distribution in Nested Systems}

Figure 1 shows the distribution of nested bar sizes, normalized by galactic diameter $\left(D_{25}\right)$. The data were divided into two groups which were split at a value which minimizes the overlap between primary and secondary bars in nested bar systems. We find that the minimal overlap between the distribution of normalized primary and secondary bar sizes for both the Seyfert and non-Seyfert galaxies occurs at a (normalized) bar length of $l_{\text {crit }} \approx 0.06$. As discussed in $\S 6.1$, $l_{\text {crit }}$ also exists in the physical bar length domain and corresponds to $\approx 1.6 \mathrm{kpc}$. Interestingly, without the triple-barred systems only one primary bar lies on the "wrong" side of the dividing line in the normalized diagram. This is the first time that such a clear separation of primary and secondary bar lengths has been shown observationally. Because our samples include all the Hubble types from S0 to Sc, the minimal overlap between the two bar classes means that our result stands regardless of the morphological class of the galaxy.

\subsubsection{Ellipticities of Nested Bars}

Figure 2 shows the ellipticity distributions of nested bars in our Seyfert and non-Seyfert subsamples. To minimize the uncertainties, we have divided all bars into two groups based on their ellipticities, $\epsilon \leq 0.45$ and $\epsilon>0.45$. The results are not overly sensitive to the exact position of this boundary. It is clear from this figure that secondary bars have a larger fraction of lower ellipticities than primary bars, both among Seyfert galaxies and among non-Seyfert galaxies. In fact, among the Seyfert double-barred systems, $80 \% \pm 13 \%$ have a higher outer bar ellipticity than inner bar ellipticity, and $71 \% \pm 17 \%$ of non-Seyfert doublebarred systems have a higher outer bar ellipticity. The simplest explanation for the prevalence of less elliptical secondary bars is that their ellipticity is diluted by the light from the galactic bulge, which is expected to be rounder than the underlying bar.

Figures $3 a, 3 b, 4 a$, and $4 b$ display the physical and normalized (divided by the $D_{25}$ diameter) bar lengths versus the deprojected ellipticities of the bars. There is a sharp increase in ellipticity toward large-scale bars, especially for Seyfert

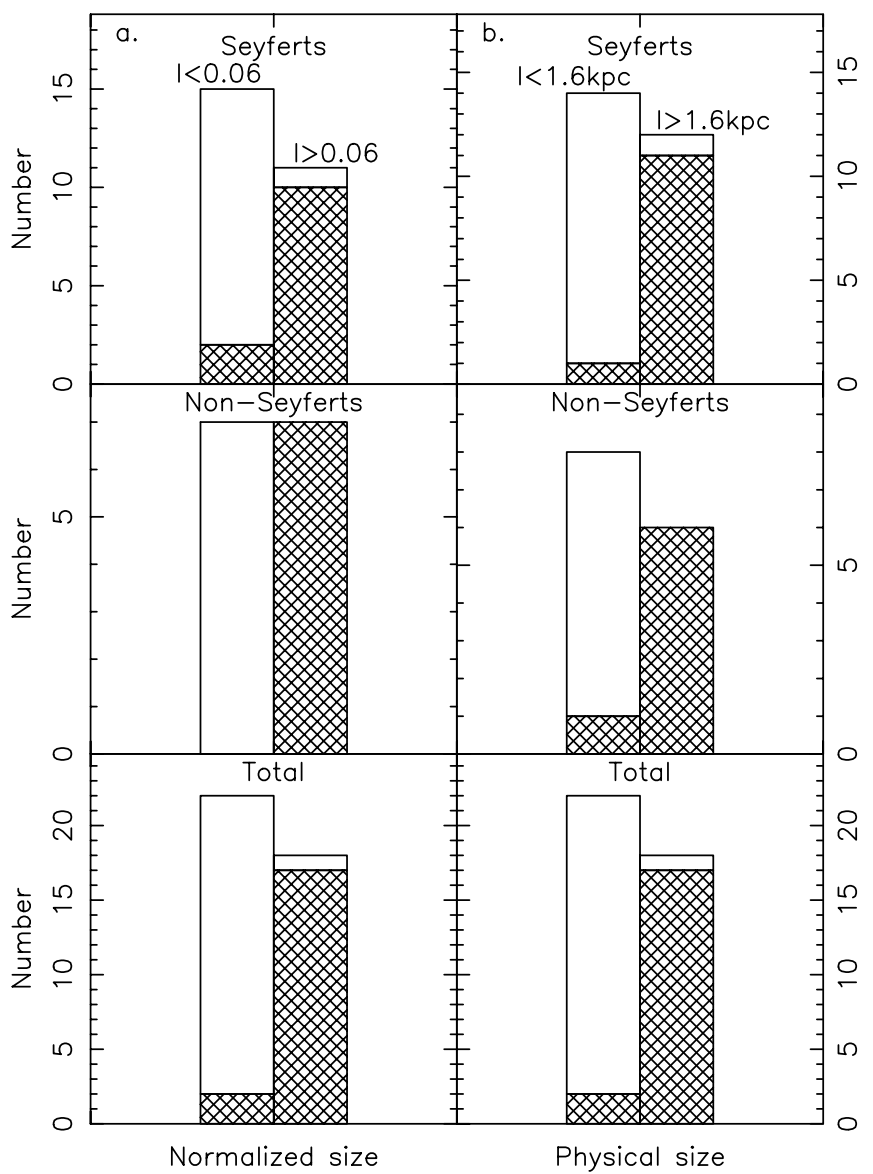

FIG. 1.-Distribution of $(a)$ normalized and $(b)$ physical primary (crosshatched) and secondary (white) bar sizes. The top panels show Seyfert galaxies, the middle panels show non-Seyfert galaxies, and the bottom panels display the totals. The bar lengths were normalized by the host galaxy diameter $D_{25}$, and the resulting values were divided into two groups, $l<l_{\text {crit }}=0.06$ and $l>l_{\text {crit }}=0.06$. In physical units the critical dividing length is $1.6 \mathrm{kpc}$. The critical dividing bar lengths $l_{\text {crit }}$ were chosen to minimize the overlap between the two groups.

galaxies. In fact, this increase is evident for all sizes in excess of $l_{\text {crit }}$ in normalized or physical bar lengths. A similar correlation, namely, that strong bars are long, has been seen earlier in later Hubble type galaxies (Martinet \& Friedli 1997; Márquez et al. 2000). Here we extend this correlation between the bar ellipticity and its length to earlier Hubble types.

\subsection{Comparison of Single and Nested Bars}

Can the single bars be meaningfully divided into two groups based on their lengths, in analogy with nested bars, where there is a fairly sharp division between secondary and primary bars in terms of their length? In other words, do the small-scale single bars exhibit properties similar to the secondary bars, which might hint about a common origin? To test this conjecture, we compared the properties of nested and single bars. Figures 3 and 4 show that if the division at $l_{\text {crit }}$ is used, only a relatively small fraction of single bars have lengths less than $l_{\text {crit }}(7 / 29$ among the Seyfert galaxies and 8/21 among the non-Seyfert galaxies).

The ratio of the number of secondary to nested (primary plus secondary) bars is $54 \%$ among the Seyfert galaxies (including the triple-barred systems) and (obviously) $50 \%$ among the non-Seyfert galaxies. While the single bars pre- 


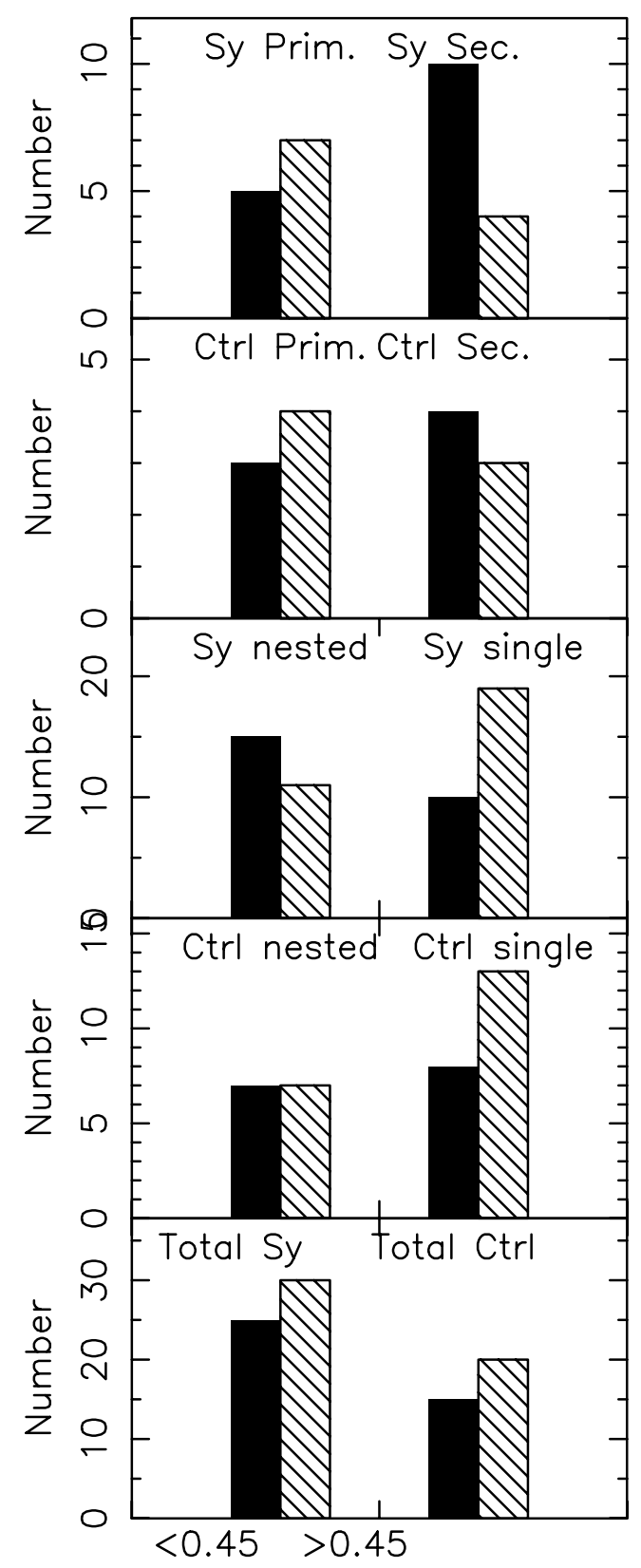

FIG. 2.-Distribution of bar ellipticities in nested and single-barred Seyfert and non-Seyfert host galaxies. Each pair of columns represents bars with $\epsilon \leq 0.45$ (black; "fatter" or "weaker" bars) and bars with $\epsilon>0.45$ (hatched; "leaner" or "stronger" bars).

dominantly lie outside $l_{\text {crit }}$ in both of our subsamples (Figs. $3 c, 3 d, 4 c$, and $4 d)$, the fraction of small-scale $\left(l<l_{\text {crit }}\right)$ single bars among all the single bars is only $24 \% \pm 8 \%$ in the Seyfert sample and $38 \% \pm 11 \%$ in the non-Seyfert sample. Thus, the fraction of small-scale single bars is significantly smaller than the corresponding fraction of secondary bars. This is the first indication that small single bars and secondary nested bars may have a different origin.

The second indication of this dissimilarity is that the ellipticity of single bars appears to be distributed differently from that of nested bars (Fig. 2). Single bars have a higher fraction of large ellipticities not only when compared to nested bars as a whole, but also when comparing single bars to primary bars alone, as can be seen in Figure 2. While the fraction of nested bars with an ellipticity less than 0.45 is

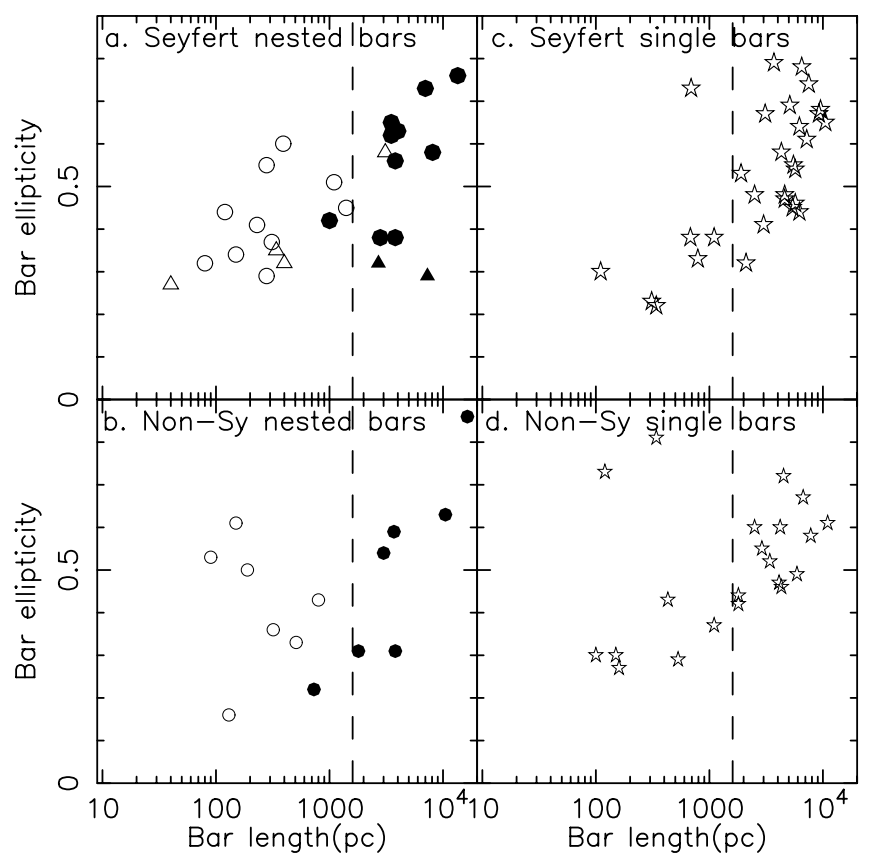

Fig. 3.-Deprojected physical bar lengths for all the bars in our samples, separately for Seyfert and non-Seyfert galaxies. The $y$-axis is the ellipticity after a two-dimensional deprojection. (a) Primary and secondary bars in the Seyfert sample. Primary bars are shown with filled circles, secondary bars with open circles. Triangles denote bars in triple-barred galaxies. (b) Same as (a), but for non-Seyfert galaxies. (c) Single bars in the Seyfert sample. $(d)$ Single bars in the non-Seyfert sample.

greater than or equal to the fraction of nested bars with an ellipticity greater than 0.45 among the Seyfert galaxies and non-Seyfert galaxies, the majority of single bars have ellipticities greater than 0.45 . This may be partly related to the fact that the majority of single bars are large-scale bars and, therefore, have larger ellipticities (§ 4.1.3).

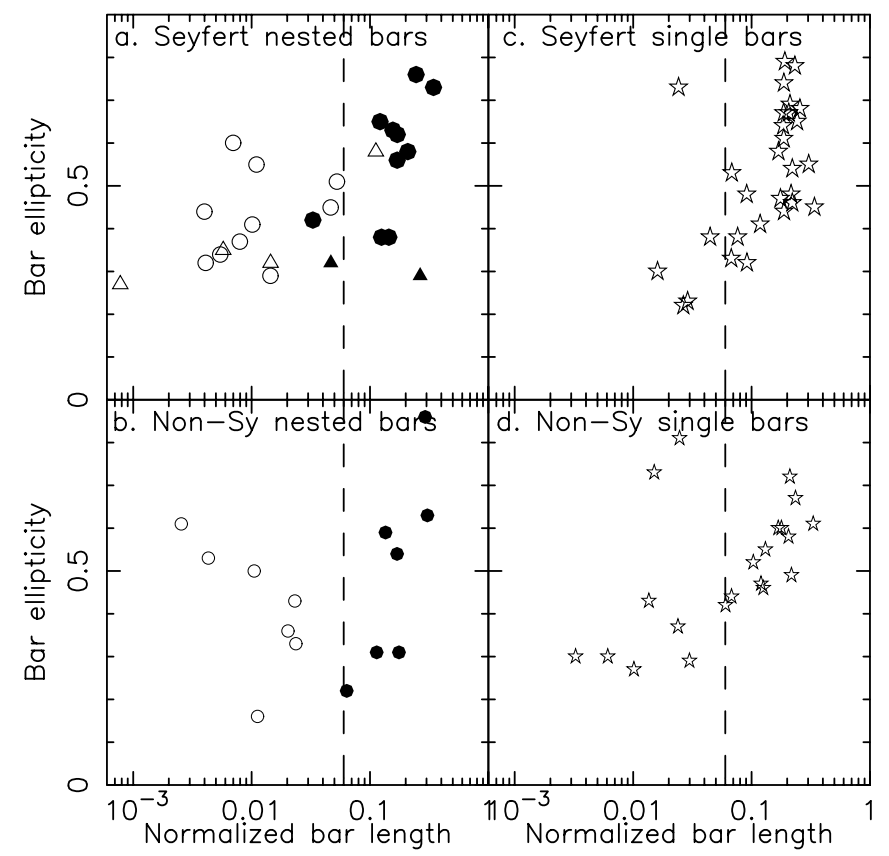

FIG. 4.- Same as Fig. 3, but now showing normalized deprojected bar lengths listed in Tables 6 and 7. 


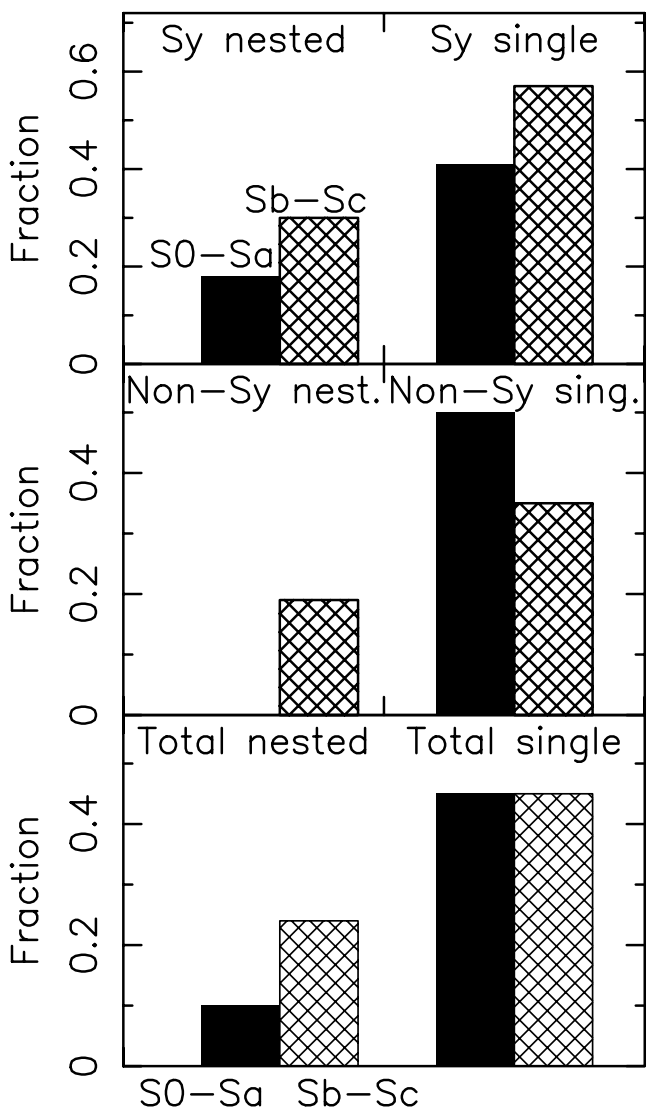

FIG. 5.-The fractions of Seyfert (top), non-Seyfert (middle), and total (bottom) galaxies with nested or single bars, divided into early $(\mathrm{S} 0-\mathrm{Sa}$; black) and late ( $\mathrm{Sb}-\mathrm{Sc}$; cross-hatched) Hubble classes. The morphological classifications were taken from NED.

Finally, we inspect the Hubble type distribution of the host galaxies of the various bar classes. Figure 5 compares the fraction of early-type ( $\mathrm{S} 0-\mathrm{Sa})$ galaxies with bars to the fraction of late-type ( $\mathrm{Sb}-\mathrm{Sc}$ ) galaxies with bars, separately for Seyfert and non-Seyfert samples, and nested and single bars. Nested bars prefer later Hubble types, whereas the single bars occupy about equal fractions among early- and late-type galaxies. Although the numbers are small, this is possibly yet another indication of the different origin of nested and single bars. The most striking result in this figure is that nested bar systems in non-Seyfert galaxies only occur in late Hubble types $(\mathrm{Sb}-\mathrm{Sc})$. This cannot be due to a lack of early-type galaxies in our non-Seyfert sample since we matched the two samples in morphological type, but of course it can be a result of small number statistics.

\section{COMPARISON OF BARS AMONG SEYFERT AND NON-SEYFERT GALAXIES}

\subsection{Bar Fraction in Seyfert and Non-Seyfert Host Galaxies}

Morphological differences between Seyfert galaxies and non-Seyfert galaxies, in general, and different bar fractions, in particular, have been sought in order to understand the fueling mechanism(s) of central stellar and nonstellar activity in active galactic nuclei (AGNs) (e.g., Adams 1977; Simkin, Sue, \& Schwartz 1980; Balick \& Heckman 1982; MacKenty 1989; see also more recent studies by Moles, Márquez, \& Pérez 1995; Ho, Filippenko, \& Sargent 1997a). The early surveys, but also the more recent ones, suffer from an absence of a properly matched control sample, from low resolution, from being conducted in the optical band where stellar bars are more difficult to detect, or from adopting the RC3 classification. When such studies were performed in the NIR, the fraction of barred galaxies increased by a factor of 2 at least compared to the fraction with SB notation in RC3, but the difference between Seyfert galaxies and non-Seyfert galaxies has been found to be statistically insignificant (Mulchaey \& Regan 1997) or marginally significant (KSP00). The newest careful NIR observations have revealed an even larger fraction of bars (Grosbøl 2001).

To detect bars, KSP00 used NIR imaging data for the CfA sample (Huchra \& Burg 1992), observed with subarcsecond resolution, and applied a set of well-defined and objective criteria. A difference between the barred fraction in Seyfert galaxies and non-Seyfert galaxies was found, but only at a $2 \sigma$ significance level (Table 1 ). Because the current sample is larger than that of KSP00, we can perform the test again, improving its statistical significance. Since we include the $H S T$ archive data, we are also able to detect smaller bars than KSP00.

Of the 56 galaxies in our current Seyfert sample, we find that 41 are barred $(73 \% \pm 6 \%)$, whereas of our 56 control galaxies, only $28(50 \% \pm 7 \%)$ are barred. We have checked the bar fractions as a function of bar length and conclude that Seyfert galaxies have more bars at practically all length scales or, at most, the bar fractions are equal. We estimate a formal significance of the result that Seyfert host galaxies are barred more often than nonactive galaxies at the $2.5 \sigma$ level, based on the quadratic combination of the uncertainties for the individual samples. Our current results are in perfect agreement with those of KSP00 (Table 1) but have a higher significance.

The overall bar fraction of $62 \% \pm 5 \%$ compares with the result of $69 \% \pm 6 \%$ as found from the NIR imaging analysis of our combined Seyfert and control samples in $\mathrm{KSP} 00$, and with other determinations in the literature, ranging from below $60 \%$ to around $75 \%$ (e.g., Mulchaey \& Regan 1997; Eskridge et al. 2000). Whereas NIR imaging surveys have led to a factor of 2 increase in the bar fraction with respect to the RC3 (SB classification), the bar fractions determined in the current work are slightly lower. This is clearly related to our choice of a conservative approach and strict criteria for bar identification (in fact, slightly more restrictive than in KSP00) and our aim for sample comparison, rather than establishing absolute numbers. Use of subjective and non-reproducible criteria, as, e.g., in Eskridge et al. (2000) and in all major galaxy catalogs, may well lead to higher bar fractions.

\subsection{Bar Strength in Seyfert and Non-Seyfert Galaxies}

We count all the bars with ellipticities greater than 0.45 as "strong" bars, although the axial ratio does not necessarily reflect the strength of a bar which is measured by the maximal ratio of tangential to radial gravitational forces (Shlosman, Peletier, \& Knapen 2000). This division gives $55 \% \pm 7 \%$ strong bars of all sizes among Seyfert galaxies and $5 \overline{7} \% \pm 8 \%$ among non-Seyfert galaxies. The same procedure applied to the secondary bars, using the same division between strong and weak bars, results in $29 \% \pm 12 \%$ strong secondary bars among Seyfert galaxies and $43 \% \pm 19 \%$ among non-Seyfert galaxies. Figure 2 contrasts the ellipticities (divided into two groups, separated by 0.45 ) between primary and secondary bars, and nested and single 
TABLE 1

Comparison of the Bar Statistics From KSP00 to the Current Paper

\begin{tabular}{|c|c|c|c|c|c|c|}
\hline \multirow[b]{2}{*}{ SAMPLE } & \multicolumn{2}{|c|}{ Size } & \multicolumn{2}{|c|}{ BAR Fraction: SEYFERT GALAXIES } & \multicolumn{2}{|c|}{ BAR FRACTION: NON-SEYFERT GALAXIES } \\
\hline & Seyfert & Non-Seyfert & $N$ & $\begin{array}{c}\text { Percentage } \\
(\%)\end{array}$ & $N$ & $\begin{array}{c}\text { Percentage } \\
(\%)\end{array}$ \\
\hline KSP00 ......... & 29 & 29 & $23 / 29$ & $79 \pm 8$ & $17 / 29$ & $59 \pm 9$ \\
\hline This paper...... & 56 & 56 & $41 / 56$ & $73 \pm 6$ & $28 / 56$ & $50 \pm 7$ \\
\hline
\end{tabular}

bars, both separately for Seyfert galaxies and non-Seyfert galaxies. It also compares all Seyfert bar ellipticities to all non-Seyfert bar ellipticities. Significantly, Seyfert galaxies always have more or at least the same number of weak bars as non-Seyfert galaxies, among secondary, primary, and single bars (Fig. 2). This reinforces the earlier results of Shlosman et al. (2000) that the bars in Seyfert galaxies are weaker than the bars in non-Seyfert galaxies.

\subsection{Comparison between Seyfert 1 and Seyfert 2 Galaxies}

Because the unified theory of AGNs (e.g., Antonucci 1993) states that Seyfert 1 and Seyfert 2 galaxies are intrinsically similar, we study the bar fractions separately for Seyfert 1 and Seyfert 2 galaxies. We group Seyfert 1-1.9 galaxies together as Seyfert 1 and compare their properties to the Seyfert 2 class (which included the Sy1h galaxies where the broad lines are seen in polarized light) and find that $16 / 23(70 \% \pm 10 \%)$ of Seyfert 1 galaxies possess at least one bar. In comparison, $25 / 33(76 \% \pm 7 \%)$ of Seyfert 2 galaxies have at least one bar. Therefore, the bar fractions are the same within uncertainties among the two Seyfert types.

We found that $13 \% \pm 7 \%$ of the Seyfert 1 galaxies and $27 \% \pm 8 \%$ of Seyfert 2 galaxies have nested bars. This difference could be due to the more luminous nuclei of Seyfert 1 galaxies hiding secondary bars or, in fact, may represent a more fundamental property of these galaxies, to be decided

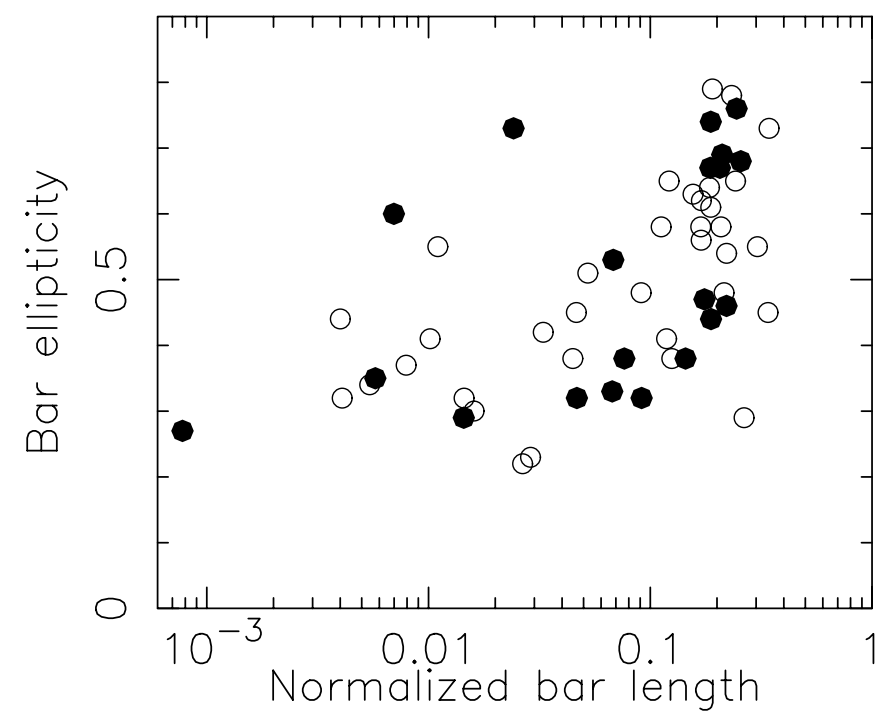

FIG. 6.- Normalized bar lengths for all the bars in the Seyfert sample. Seyfert 1 bars are shown with filled circles and Seyfert 2 bars with open circles. The $x$-axis is the normalized bar length after deprojection (normalized to the galaxy diameter, tabulated in Tables 6 and 7), and the $y$-axis is the ellipticity after a two-dimensional deprojection. in larger samples. Lastly, Figure 6 shows that distributions of the normalized lengths of the Seyfert bars (normalized by the galaxy diameter) versus ellipticity separately for Seyfert 1 and Seyfert 2 galaxies are similar.

\section{DISCUSSION}

\subsection{Nested and Single Bar Properties}

The observed nested bars in our samples of Seyfert and non-Seyfert galaxies reveal an intriguing property: the existence of a critical physical length, $\approx 1.6 \mathrm{kpc}$, which separates the primary and the secondary bars, resulting in a clear bimodal size distribution with only little overlap. Moreover, when the bar sizes are normalized to those of the respective host galaxies, the overlap between the two bar species is further reduced. To illustrate this effect, we have constructed Figure 7, where the physical sizes of deprojected bars are shown versus $D_{25}$. We note that in this figure the primary bar sizes exhibit a roughly linear correlation with the parent galaxy sizes (the linear correlation coefficient is 0.66 , and the probability that this is achieved by uncorrelated points is less than $1 \%$ ). The slope of this correlation is finite and nonzero. On the other hand, the secondary bar sizes are limited by an upper boundary. This can be interpreted as a linear correlation with a zero slope, or, in other words, the sizes of these bars are independent from the sizes of their host galaxies. The importance of this result can be inferred from the fact that only in this case the normalized

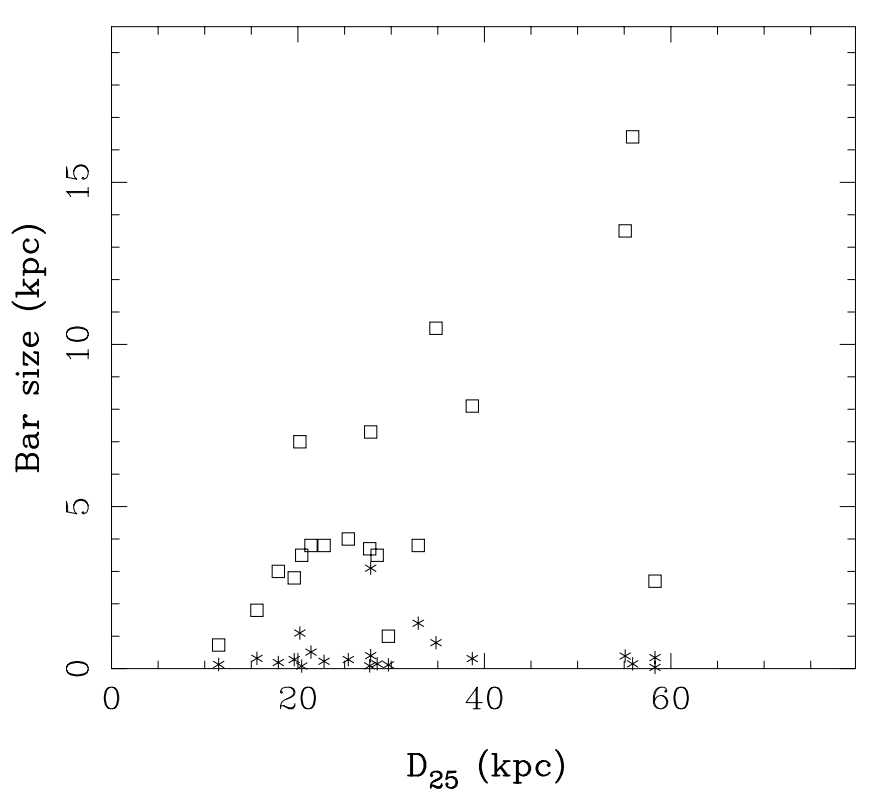

FIG. 7.-Primary (open squares) and secondary (asterisks) bar sizes vs. 
(to $D_{25}$ ) bar lengths will preserve the identity of both bar groups and there will be no further mixing between the primary and secondary bars in the normalized size space, as shown in Figures 3 and 4. If, for example, both types had a linear correlation with $D_{25}$ where both slopes were nonzero, the two bar groups (primary and secondary bars) would be separated in physical space but mixed in the normalized space.

We suggest a simple and attractive explanation for the presence of a bimodal distribution of bar sizes both in the physical and in the normalized space, related to the property that secondary bars are limited to within $\sim 1.6 \mathrm{kpc}$ in their physical size. The linear correlation of the primary bar size with galaxy size means that these bars extend to a fixed number of radial scale lengths in the disk. The absence of such a correlation for the secondary bars, together with their limited range of sizes, hints at a different physical nature of formation and dynamics compared to the primary bars.

As discussed in $\S 2$, numerical simulations of nested bars show that the secondary bars are confined to the region within the ILRs of the primary bars. These ILRs develop close to the radius of the rotation velocity turnover (or a substantial bump in the rotation curve), which is generally located just outside the bulge (at least for early Hubble types), or more basically, where the mass distribution in the inner galaxy switches from three-dimensional to twodimensional with increasing radius. In a hypothetical case of a plane-parallel and uniform galactic disk, this happens at $r \sim \Delta z$, where $\Delta z$ is the thickness of the disk. For a realistic surface density distribution in the disk and in the presence of a bulge, one can use $\sim 1-2 \mathrm{kpc}$ as a reasonable estimate for the position of the ILRs (in fact, of the outer ILR). For the early Hubble type galaxies ( $\mathrm{S} 0-\mathrm{Sb}$ ), this leads to the appearance of an ILR at about the bulge radius. For the later Hubble type galaxies or early types with small bulges, the height of the disk becomes comparable to the radius of the disk at a point where the two-dimensional disk approximation breaks down. Further evidence for a constant normalized position of the ILR in disk galaxies of all sizes is provided by Athanassoula \& Martinet (1980) and Martin (1995), who found a linear correlation between the large-scale bar and bulge sizes.

If primary and secondary bars had a similar formation and evolution history, one would expect a linear correlation between the secondary bar length and $D_{25}$ simply because of the observed correlations between the disk, large-scale bar, and bulge sizes. However, this correlation is clearly ruled out by our data. A possible resolution of this discrepancy is as follows.

Large-scale bars are known to extend to about $0.83 \pm 0.12$ of their corotation radii, an empirical rule based on the shapes of their offset dust lanes (Athanassoula 1992). However, the secondary bars are not expected to follow this rule or to possess offset dust lanes (Shlosman \& Heller 2002). Because a large degree of dissipation is involved in forming these small bars, their size can be much smaller than their corotation radius (which is also the ILR of the primary bar). This particular property of secondary bars is expected to destroy any correlation between their size and that of the parent galaxy.

We have compiled a sample of 62 galaxies with nuclear rings (mostly from Buta \& Crocker 1993) and determined their normalized size distribution (see Fig. 8). This distribu-

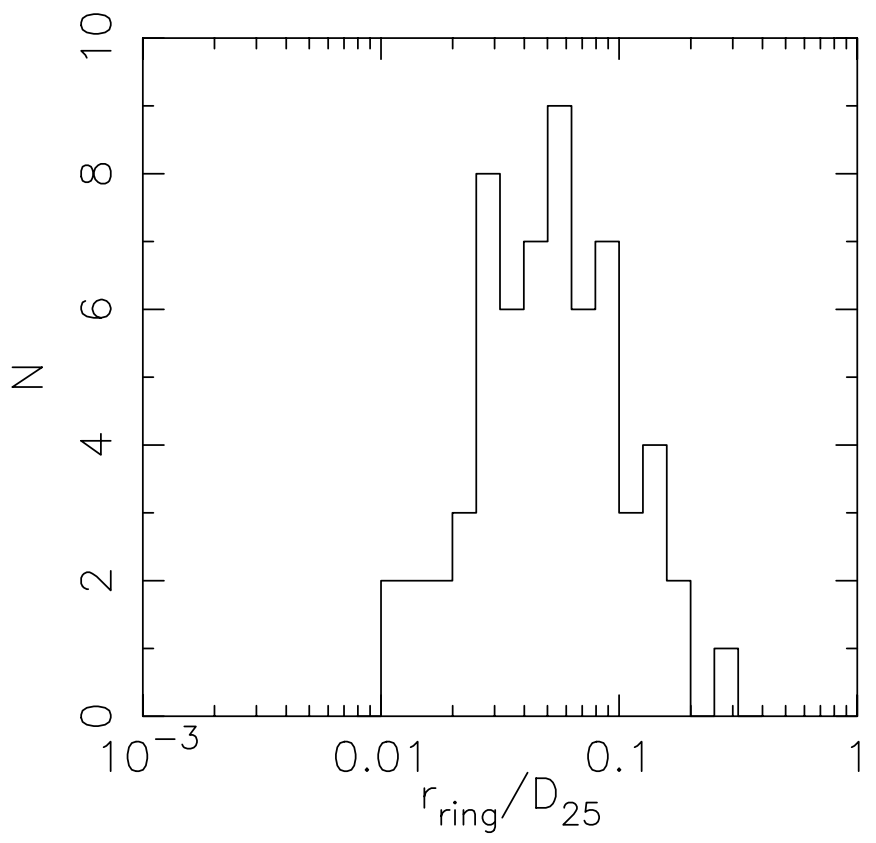

FIG. 8.-Number distribution of normalized nuclear ring diameters. Data were mostly taken from Buta \& Crocker (1993), but we added a few "famous" nuclear ring galaxies.

tion peaks at $r_{\text {ring }} / D_{25}=0.06$, supporting our view that it acts as the dynamical separator between secondary and primary bars. This result is consistent with our claim that the secondary bar lengths are limited by the size of the ILR, since nuclear rings are associated with the ILRs (e.g., Schwarz 1984; Combes \& Gerin 1985; Knapen et al. 1995a).

\subsection{Comparison with Earlier Studies of Secondary Bars}

6.2.1. Ground-based Studies

Previous ground-based studies (e.g., Shaw et al. 1995; Jungwiert et al. 1997; Erwin \& Sparke 1999b) have found secondary bars in $20 \%-25 \%$ of their sample galaxies. Only a fraction of their secondary bars fulfill our bar criteria. In addition, there are biases in these samples, e.g., Shaw et al. (1995) and Erwin \& Sparke (1999b) start with samples where the outer bar is well detected. Shaw et al. (1995) define as secondary bars those NIR isophote twists which occur inside the minor axis width of the large-scale bar and where the secondary bar is not aligned with the large-scale bar. They found at most seven $(24 \%)$ such cases in a sample of 29 relatively face-on galaxies. Ellipticity and position angle profiles were given only for five of these objects. Of these only one, NGC 4321, fulfills our criteria for a bar.

Jungwiert et al. (1997) considered a sample of 56 galaxies with inclinations less than $75^{\circ}$. We note that it may be exceedingly difficult to find bars at such high inclinations. Jungwiert et al. (1997) have found 17 galaxies (30\%) with two triaxial (and therefore possibly double-barred) structures. Of these, only eight are secondary bars according to our criteria. In addition, they find bar signatures (ellipticity peaks with constant position angles) in the nuclear regions (less than $1 \mathrm{kpc}$ ) of three SA galaxies. Deprojection effects were also studied and accounted for in a simple twodimensional approximation. Erwin \& Sparke (1999b) find five $(23 \%)$ definitely double-barred galaxies in a sample of 22 barred galaxies by fitting ellipses to the isophotes. Only 
two profiles are given, and of these only one fits our bar criteria. In addition, they find another five possible doublebarred systems. One of their definitely barred galaxies, NGC 2681, is claimed to be triple barred.

\subsubsection{Previous HST Studies of Small Bars}

Recent papers by Regan \& Mulchaey (1999) and Martini \& Pogge (1999) suggest that nuclear bars may not be important for fueling AGN activity because nuclear bars were found in only a small minority of their samples. Specifically, Regan \& Mulchaey (1999) claim that the Seyfert galaxies Mrk 573 and Mrk 1066 do not have nuclear bars, based on their nuclear dust morphology. However, we have found subkiloparsec bars in both of these galaxies. On the other hand, Regan \& Mulchaey (1999) claim that NGC 5347 and NGC 7743 have nuclear bars, whereas we have not detected them by ellipse fitting. Furthermore, Martini et al. (2001) discuss the role of secondary bars in the fueling of the nuclear activity in disk galaxies without invoking a matched control sample of nonactive galaxies.

One should be aware of the caveats associated with the Regan \& Mulchaey (1999) result. First, they looked for straight segments of dust lanes in the circumnuclear region. Such dust lanes are usually seen on the leading side of large-scale bars. However, the physical conditions in the circumnuclear region are known to differ from those at kiloparsec scales, and there is no reason why gasdynamics and stellar dynamics should be identical as well. Shlosman \& Heller (2002) have analyzed the gas flow in secondary bars and found that gas flow in the secondary bars differs from that in the primary bars as a result of a time-dependent potential, fast rotation, specifics of gas crossing the bar-bar interface, and other reasons related to secondary bar formation. No offset dust lanes form under these conditions.

\subsection{Excess of Bars among Seyfert Galaxies \\ 6.3.1. Comments on Bar Detection}

The main result from the comparison of Seyfert and nonSeyfert galaxy bars that emerges from our study is that Seyfert galaxies have more bars on almost any length scale. Evidently on the largest scales stellar bars (spontaneous and induced) are so frequent that when taken together with oval distortions of disks, they become nearly universal. Under these circumstances it is clear that large-scale bars are an important but not a sufficient factor to fuel the nuclear activity in AGNs, as was already pointed out by Shlosman et al. (1989). It has been established beyond doubt during the last decade that large-scale bars are efficiently channeling gas toward the central kiloparsec and induce starburst activity there, mainly in the form of nuclear rings. However, this does not explain the fate of the inflowing gas at even smaller scales, between a few hundred parsecs and $1 \mathrm{pc}$. The mere existence of secondary bars on these small spatial scales hints that gravitational torques are important here, but the exact role of secondary bars in the fueling hierarchy as well as the details of their formation and evolution are obscure. Here we focus on the properties of stellar bars in the NIR, but one should not forget that at least one additional factor must play a crucial role in differentiating between Seyfert and non-Seyfert hosts, the availability of digestible fuel, and therefore the knowledge of dynamics of the self-gravitating gas in the background potential of nested bars is of prime importance (Shlosman et al. 1989).
Given the poor record of detecting large-scale bars in the optical, the bulge light dilution of secondary bar isophotes in the NIR, effects of dust and star formation within the central kiloparsec (even in the NIR), and finally our conservative approach to bar detection, it is not surprising that in this paper we have detected only modest bar fractions in disk galaxies. We have found that stellar dynamics cannot be reliably traced even by the use of NIR surface photometry. This should be taken into account while assessing the significance of recent studies in this field, such as the work of Martini et al. (2001). Nevertheless, this fraction by far exceeds the fraction of galaxies classified as "SB" in any optical catalog, e.g., the RC3.

It is known, for example, that Seyfert 2 galaxies, which comprise the large majority of our Seyfert sample, are dustier and include more star formation (e.g., González Delgado, Heckman, \& Leitherer 2001). The deprojection procedure will introduce additional uncertainties in the bar axial ratios. On the other hand, there is probably not enough dust present even in the inner regions of spirals to hide many bars in the $H$ band. Giovanelli et al. (1994) showed that in the Cousins $I$ band the central optical depth is smaller than 5. Assuming the Galactic extinction law (Rieke \& Lebofsky 1985), this corresponds to an optical depth of 1.6 in $H$. Since this value goes down rapidly with decreasing inclination, most galaxies have central optical depths in $H$ smaller than 1 mag (in agreement with Peletier et al. 1995), implying that it is hard to hide bars in the circumnuclear regions of disk galaxies. Possibly the largest unknown in the overall picture of nested bars is the lifetime of secondary bars which will affect directly their observed frequency.

One should also remark on the deprojected ellipticity distribution of bars on all spatial scales. We find that the ellipticity distribution peaks at about $\epsilon \sim 0.4-0.5$. The numbers decline for larger ellipticities, as well as for smaller ellipticities. The same trend is preserved if only relatively face-on galaxies are counted, say, with axial ratios greater than 0.85 . No theoretical explanation exists presently for this behavior.

\subsubsection{Galaxy Interactions: Induced and Spontaneous Bars in Disk Galaxies}

The effects of interactions on inducing the formation of stellar bars have not been exhaustively studied. Noguchi (1988) and Salo (1991) suggested that flybys induce stellar bars when tidal forces exceed about $10 \%$ of the unperturbed radial force and when the encounter is prograde. There is no indication so far that the gas flow pattern differs between induced and spontaneous (i.e., those formed as a result of bar instability) bars. Because such tidally induced bars are indistinguishable from bars formed in a bar instability, and because we aim at a detailed comparison between the occurrence and properties of bars in Seyfert and non-Seyfert control galaxies, irrespective of their origin, our strategy is not to exclude interacting galaxies, apart from the strongly distorted ones. In other words, for the purpose of understanding the morphological differences which may be responsible for fueling the central activity in disk galaxies, it is irrelevant which type of bar is hosted by the galaxy. One needs only to exclude the strongly interacting galaxies which are heavily distorted and, therefore, complicate the bar identification.

The criticism by Márquez et al. (2000) of the KSP00 
results by implying that the excess of bars among Seyfert hosts is due to the inclusion of galaxies that may be undergoing gravitational interaction is therefore not justified. Moreover, it is unclear what can be achieved by following the Márquez et al. (2000) procedure, which reduced the KSP00 sample to 13 (Seyfert) and 11 (control) galaxies by rejecting all galaxies that have companions within a cylindrical volume of $0.4 \mathrm{Mpc}$ in projected radius and $2 \times 500$ $\mathrm{km} \mathrm{s}^{-1}$ in $c z$ (distance along the line of sight, corresponding to $6.7 \mathrm{Mpc}$ when assuming a Hubble flow with $H_{0}=75 \mathrm{~km}$ $\mathrm{s}^{-1} \mathrm{Mpc}^{-1}$ ). Clearly, the few remaining galaxies in the sample are insufficient to allow any meaningful conclusions on barred fractions in different samples. The derived numbers are not statistically significant and cannot be interpreted as evidence against higher bar fractions among Seyfert galaxies.

We note that although Seyfert activity occurs in interacting and merging galaxies, there is no significant evidence for an excess of companions to Seyfert galaxies as compared to nonactive control galaxies (e.g., Fuentes-Williams \& Stocke 1988; de Robertis, Yee, \& Hayhoe 1998). Earlier work by, e.g., Adams (1977) and Dahari (1984) was plagued by poor control sample selection. This implies that there is no a priori reason to reduce samples of Seyfert and nonactive galaxies artificially by excluding all galaxies with companions; the only effect of such an operation is the reduction of the numbers of galaxies in both samples in equal amounts, which effectively corresponds to an increase in the statistical uncertainty of the final result.

In order to check the statements above with the galaxies in our samples, we have used the Lyon-Meudon extragalactic database (LEDA) to find companions to all our sample galaxies. As a first step we used the same criteria as Márquez et al. (2000) to find those sample galaxies which have companions within $400 \mathrm{kpc}$ in radius and within \pm 500 $\mathrm{km} \mathrm{s}^{-1}$ in $c z$. We found that 34 (out of 56) or $61 \% \pm 7 \%$ of our Seyfert galaxies and 46 (out of 56 ) or $82 \% \pm 5 \%$ of our control galaxies in fact have companions within such a volume. We also found, though, that this fraction reaches almost $100 \%$ for the nearest galaxies in our sample, in line with experience that most if not all galaxies will have companions of some size (e.g., the Milky Way, M31).

We refined our search in the second step, where we impose the additional criterion that companion galaxies may not be fainter than the sample galaxy by $\Delta B_{T}=1.5$ mag. This limit is somewhat arbitrary but ensures that the companion to M51, NGC 5195, is included under these criteria. We call the sample galaxies with such bright companions "interacting" although they may not be so at a level which distorts their appearance. We find that in both the Seyfert and the control sample, 23 out of $56(41 \% \pm 7 \%)$ of sample galaxies are interacting, while the remaining 33 $(59 \% \pm 7 \%)$ are not. We thus find no evidence for a difference between the Seyfert and control sample in terms of bright companions, in agreement with the findings of Schmitt (2001), but do find that our control galaxies have (faint) companions significantly more often than the Seyfert galaxies do. This cannot be an effect of closer distance of the control galaxies since we matched them in distance to the Seyfert sample.

The important question in relation to the current paper is whether the presence of these companions, bright or faint, is related to the presence of a bar (as claimed by Márquez et al. 2000). The bar fractions, and associated uncertainties from Poisson statistics, are given in Table 2 for all the subsamples as defined above (Seyfert and control, with and without faint and bright companions). The conclusion from these numbers is that the bar fraction among our sample galaxies is completely independent of the presence of faint, or bright, companions. This conclusion does not contradict the earlier study by Elmegreen, Elmegreen, \& Bellin (1990), which showed that there is an excess of bars among earlytype galaxies in strongly interacting pairs. Elmegreen et al. (1990) used the "SB" classification in optical catalogs instead of ellipse fitting to find bars, and their sample consisted of much closer galaxy pairs. In summary, we have addressed the kind of criticism raised by Márquez et al. (2000) by demonstrating that the presence of a bar in our sample galaxies is not related to the presence of companions.

\section{CONCLUSIONS}

We have taken advantage of the high spatial resolution offered by the $H S T$ and the large database of disk galaxies of Hubble types S0-Sc in the HST archive, complemented by ground-based NIR and optical images of the outer disks, to examine the numbers and properties of bars in 56 Seyfert and 56 matched non-Seyfert galaxies. We emphasize that our results still suffer from small number statistics and, therefore, must be confirmed by a study of larger samples. The use of adaptive optics on ground-based telescopes and further imaging by the HST promises to increase the number of suitable Seyfert and non-Seyfert galaxies substantially in the near future. This will allow the determi-

TABLE 2

Bar Fractions and Presence of Faint or Bright Companions, for Seyfert and Non-Seyfert Samples

\begin{tabular}{|c|c|c|c|c|}
\hline \multirow[b]{2}{*}{ SAMPLE } & \multicolumn{2}{|c|}{ BAR Fraction: SEYFERT GALAXIES } & \multicolumn{2}{|c|}{ BAR FraCtION: NON-SEYFERT GALAXIES } \\
\hline & $N$ & $\begin{array}{c}\text { Percentage } \\
(\%)\end{array}$ & $N$ & $\begin{array}{c}\text { Percentage } \\
(\%)\end{array}$ \\
\hline Overall ........... & $41 / 56$ & $73 \pm 6$ & $28 / 56$ & $50 \pm 7$ \\
\hline No companions ${ }^{\mathrm{a}}$.. & $16 / 22$ & $73 \pm 9$ & $5 / 10$ & $50 \pm 16$ \\
\hline $\operatorname{Companion}(\mathrm{s})^{\mathrm{a}} \ldots$ & $25 / 34$ & $74 \pm 8$ & $23 / 46$ & $50 \pm 7$ \\
\hline Not interacting ${ }^{\mathrm{b}} \ldots \ldots$ & $24 / 33$ & $73 \pm 8$ & $16 / 33$ & $48 \pm 9$ \\
\hline Interacting ${ }^{b} \ldots \ldots \ldots \ldots$ & $17 / 23$ & $74 \pm 9$ & $13 / 23$ & $57 \pm 10$ \\
\hline
\end{tabular}

\footnotetext{
${ }^{\mathrm{a}}$ Indicates presence or absence of companion galaxies within $400 \mathrm{kpc}$ in radius and within $\pm 500 \mathrm{~km} \mathrm{~s}^{-1}$ in $c z$.

${ }^{\mathrm{b}}$ Idem, but additionally companion galaxy may not be fainter by $B_{T}=1.5 \mathrm{mag}$ than the sample galaxy under consideration for the latter to be qualified as "interacting" (see text).
} 
nation of the exact dynamical role that nested bars play in the evolution of disk galaxies and the fueling of central activity, stellar and nonstellar.

Our results are not sensitive to the exact definition of a bar. To verify this, we have varied the cutoff ellipticity of the bar within $20 \%$ and the range of allowed variation in the bar position angle within $50 \%$. Those resulted in insignificant changes in the bar statistics. Our main results are listed in the following:

1. We find that primary and secondary bar sizes, both physical and normalized by the galaxy diameter $D_{25}$, show a bimodal distribution with little overlap between the two groups. The separating value, when normalized by the galaxy diameter $D_{25}$, is around 0.06 (i.e., 0.12 of the corresponding galaxy radius). In a physical space, the dividing length between the primary and secondary bars is about 1.6 $\mathrm{kpc}$. We identify these critical values with the location of the ILRs, dynamical separators between the nested bars. The ILRs are expected to form where the gravitational potential of the inner galaxy switches from three-dimensional to twodimensional. This happens at the bulge-disk interface, or alternatively, where the disk thickness becomes comparable to its radius.

2. The distribution of nuclear ring sizes (radii) obtained from the literature was found to peak at the same normalized critical length of 0.06 . A nuclear ring is considered to be a clear indicator of the ILR in the disk and is formed just interior to this resonance in all numerical simulations of gas flows in barred galaxies. We interpret the correlation between the nuclear ring sizes and the critical value of 0.06 in nested bars as an additional strong indication in favor of the crucial roles the ILRs play in the dynamics of these systems.

3. Primary bars in nested systems show a linear correlation with the size of the host galaxy disk, extending to a fixed number of disk scale lengths.

4. We find that the secondary bar sizes do not correlate with the disk sizes, primary bar sizes, or the critical size of 0.06. As a corollary, these bars do not correlate with the radii of the primary ILRs (which coincide with the secondary bar corotation radii). This is contrary to the behavior of primary (and single) bars which are known to extend to within $0.83 \pm 0.12$ of their corotation radii.

5. Within the nested bars, the secondary bars have smaller ellipticities than the primary bars. This can be simply explained by noting that the secondary bars lie within the galactic bulges which dilute the observed small bar ellipticities, making them rounder.

6 . Both the single and primary bars show a correlation between the ellipticity and the length of the bar. This result has been reported earlier for late-type disk galaxies, but it is extended here for galaxies which range in Hubble type from S0 to Sc.

7. A relatively small fraction of single bars are shorter than the critical value of 0.06 , the boundary between the primary and secondary bars in nested systems. Single bars also have higher average ellipticities than nested bars and a different distribution in morphological types. Although the numbers are small, this raises the interesting possibility that single bars have a different formation mechanism.

8. The comparison of bar numbers between Seyfert and non-Seyfert galaxies shows that Seyfert galaxies have an excess of bars, namely, $73 \% \pm 6 \%$ of Seyfert galaxies have at least one bar, against only $50 \% \pm 7 \%$ of non-Seyfert galaxies. The statistical significance of this result is at the $2.5 \sigma$ level and confirms and strengthens the result of KSP00 which was based on smaller samples.

9. We confirm numerically that within our samples the presence of companions, even bright ones, near a galaxy bears no relation to the presence of a bar in that galaxy.

10. Seyfert galaxies have thicker (in the sense of axial ratio $b / a$ ) bars on average than the non-Seyfert galaxies, no matter how the comparison is made (among primary bars, secondary bars, single bars, or as a function of the host galaxy Hubble type). We thus confirm our earlier result of a deficiency of thin bars among Seyfert galaxies in a study of the CfA sample of Seyfert galaxies and a matched control sample of non-Seyfert galaxies.

11. We find a difference in the fraction of nested bars among Seyfert 1 galaxies $(13 \% \pm 7 \%)$ and Seyfert 2 galaxies $(27 \% \pm 8 \%)$. This effect can be most probably explained by the very luminous Seyfert 1 nuclei.

Overall, we find that NIR isophote fitting, a highly reliable method of detecting large-scale stellar bars, shows difficulties when applied to subkiloparsec bars. The main difficulty comes from localized and distributed sites of dust extinction and bright stars within the central kiloparsec. This results in a substantial underestimate of bar fraction.

We are grateful to Lia Athanassoula, Albert Bosma, Tim Heckman, and Clayton Heller for illuminating discussions. We thank Françoise Combes, Zsolt Frei, Isabel Márquez, and Kartik Sheth for providing us with some images. S. L. thanks Richard Hook for advice on the use of the CPLUCY deconvolution routine and S. Jogee for bringing to our attention a flaw in the deprojection program which we subsequently corrected. Our work is based on observations with the NASA/ESA $H S T$, obtained from the data archive at the STScI, which is operated by the Association of Universities for Research in Astronomy, Inc., under NASA contract NAS 5-26555. Our research is partly based on observations made with ESO telescopes at La Silla. The J. Kapteyn and W. Herschel Telescopes are operated on the island of La Palma by the Isaac Newton Group (ING) in the Spanish Observatorio del Roque de los Muchachos of the IAC. Data were retrieved from the ING archive. This research has made use of the NASA/IPAC Extragalactic Database (NED), which is operated by the JPL, Caltech, under contract with NASA. We have made use of the LEDA database. ${ }^{5}$ The DSSs were produced at the STScI under US Government grant NAG W-2166. The images of these surveys are based on photographic data obtained using the Oschin Schmidt Telescope on Palomar Mountain and the UK Schmidt Telescope. This publication makes use of data products from 2MASS, which is a joint project of the University of Massachusetts and IPAC/Caltech, funded by NASA and NSF. I. S. acknowledges support from NASA grants HST AR-07982.01-96A, GO-08123.01-97A, NAG 5-3841, NAG 5-10823, and WKU-522762-98-6 and thanks the organizers of INAOE workshop on "Disk Galaxies: Kinematics, Dynamics, and Perturbations" for supporting a prolonged visit during which this work was finalized.

\footnotetext{
${ }^{5}$ http://leda.univ-lyon1.fr.
} 


\section{APPENDIX A}

\section{GRAPHICAL AND TABULAR REPRESENTATION OF SAMPLE MATCHING}

The details of how we matched the Seyfert galaxy sample properties to those of a control sample of non-Seyfert galaxies were explained in $\S 3.2$. In this appendix we show a tabular and graphical representation of the galaxies in the various bins of the four quantities that were matched, distance, absolute $B$ magnitude, axial ratio, and morphological type, in Table 3 and Figure 9, respectively. The general properties of the galaxies in the adopted samples are given in Tables 4 and 5.

TABLE 3

Match of the Seyfert and Non-Seyfert Galaxy Samples with Respect to Absolute $B$ Magnitude, Distance, Axial Ratio, and MORPHOLOGICAL TYPE

\begin{tabular}{|c|c|c|c|c|c|c|c|c|c|c|c|c|c|c|c|}
\hline $\begin{array}{c}\text { Type } \\
\text { (1) }\end{array}$ & $\begin{array}{c}\operatorname{Mag}_{1} \\
\text { (2) }\end{array}$ & $\begin{array}{c}\mathrm{Mag}_{2} \\
\text { (3) }\end{array}$ & $\begin{array}{c}\text { Dist }_{1} \\
\text { (4) }\end{array}$ & $\begin{array}{c}\text { Dist }_{2} \\
\text { (5) }\end{array}$ & $\begin{array}{c}b / a_{1} \\
(6)\end{array}$ & $\begin{array}{c}b / a_{2} \\
(7)\end{array}$ & $\begin{array}{c}b / a_{3} \\
(8)\end{array}$ & $\begin{array}{c}b / a_{4} \\
(9)\end{array}$ & $\begin{array}{l}b / a_{5} \\
(10)\end{array}$ & $\begin{array}{c}\mathrm{T}_{1} \\
(11)\end{array}$ & $\begin{array}{c}\mathrm{T}_{2} \\
(12)\end{array}$ & $\begin{array}{c}\mathrm{T}_{3} \\
(13)\end{array}$ & $\begin{array}{c}\mathrm{T}_{4} \\
\text { (14) }\end{array}$ & $\begin{array}{c}\mathrm{T}_{5} \\
(15)\end{array}$ & $\begin{array}{c}T_{6} \\
(16)\end{array}$ \\
\hline Seyfert ........... & 32 & 24 & 25 & 31 & 10 & 10 & 16 & 10 & 10 & 13 & 9 & 9 & 12 & 6 & 7 \\
\hline
\end{tabular}

Note.-Col. (1): Type of galaxy. Col. (2): Number of galaxies with absolute $B$ magnitude less than -20.4. Col. (3) Number of galaxies with absolute $B$ magnitude $\geq-20.4$. Col. (4): Number of galaxies with distance smaller than $28 \mathrm{Mpc}$. Col. (5): Number of galaxies with distance larger than $28 \mathrm{Mpc}$. Col. (6): Number of galaxies with $b / a<0.6$. Col. (7): Number of galaxies with $0.6 \leq b / a<0.7$. Col. (8): Number of galaxies with $0.7 \leq b / a<0.8$. Col. (9): Number of galaxies with $0.8 \leq b / a<0.9$. Col. (10): Number of galaxies with $b / a \geq 0.9$. Col. (11): Number of galaxies with morphological $\mathrm{T}$ type $\mathrm{T}<0.1$. Col. (12): Number of galaxies with morphological $\mathrm{T}$ type $0.1 \leq \mathrm{T}<1.1$. Col. (13): Number of galaxies with morphological $\mathrm{T}$ type $1.1 \leq \mathrm{T}<2.1$. Col. (14): Number of galaxies with morphological $\mathrm{T}$ type $2.1 \leq \mathrm{T}<3.1$. Col. (15): Number of galaxies with morphological $\mathrm{T}$ type $3.1 \leq \mathrm{T}<4.1$. Col. (16): Number of galaxies with morphological $\mathrm{T}$ type $\mathrm{T} \geq 4.1$.
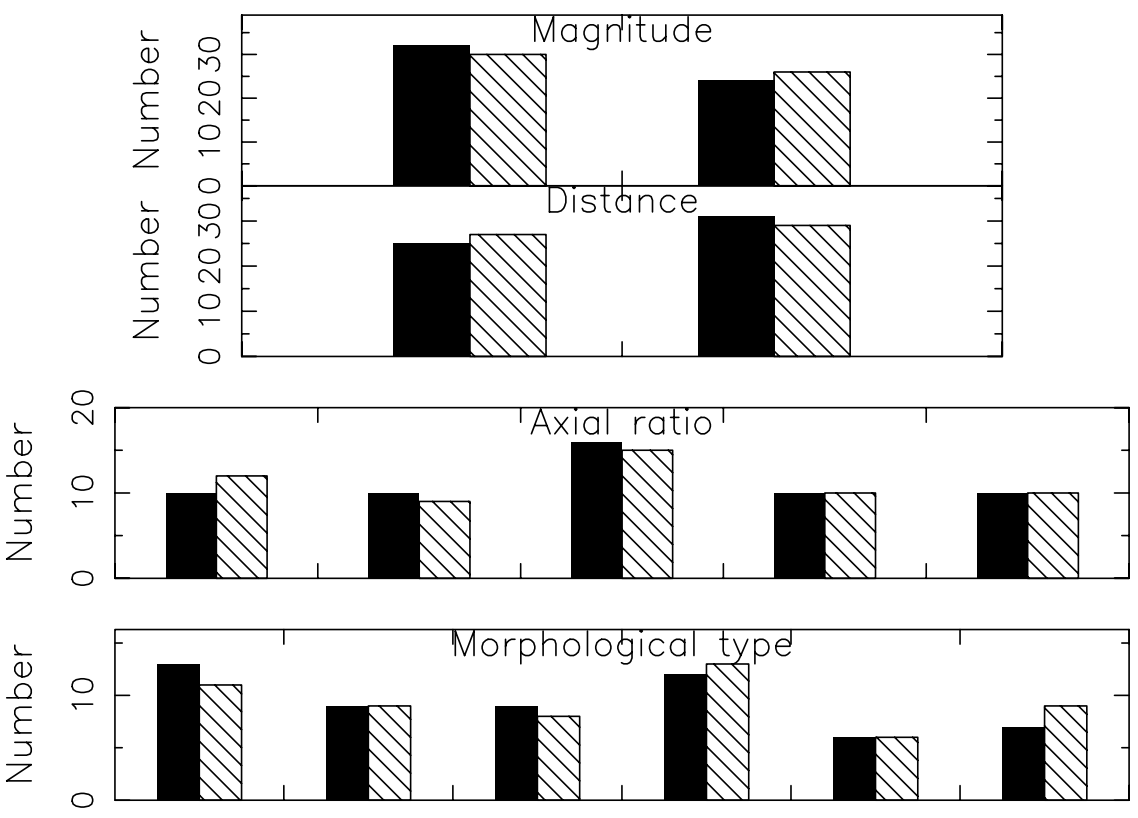

FIG. 9.-Matching of the Seyfert and non-Seyfert samples with respect to four different properties. The absolute $B$ magnitude separator is -20.4 mag. The distance separator is $28 \mathrm{Mpc}$. The axial ratio bins are separated by $0.6,0.7,0.8$, and 0.9 . Finally, the morphological $\mathrm{T}$ types are separated by $0.1,1.1,2.1,3.1$, and 4.1. The borders between the bins were selected so that we have approximately equal numbers in each bin. The Seyfert galaxies are shown with black columns and non-Seyfert galaxies with hatched columns.

\section{APPENDIX B}

\section{BAR PROFILES AND PROPERTIES}

The details of bar detection were described in $\S 3.4$. Here we present the ellipse fits covering the whole galaxies in Figures 10 (Seyfert galaxies) and 11 (non-Seyfert galaxies), showing the ellipticities and position angles of the fitted ellipses as a function of radius. The lengths and ellipticities of the detected bars are tabulated in Tables 6 (Seyfert galaxies) and 7 (non-Seyfert galaxies), together with $1 \mathrm{kpc}$ in arcseconds and the galaxy diameter $D_{25}$ in arcseconds. 
TABLE 4

Properties of Seyfert Galaxy SAmple

\begin{tabular}{|c|c|c|c|c|c|c|c|c|c|}
\hline $\begin{array}{l}\text { Galaxy } \\
\text { (1) }\end{array}$ & $\begin{array}{c}H S T \text { Source } \\
\text { (2) }\end{array}$ & $\begin{array}{l}\text { Other Source } \\
\text { (3) }\end{array}$ & $\begin{array}{c}\text { Classification } \\
\text { (4) }\end{array}$ & $\begin{array}{c}\mathrm{T} \\
(5)\end{array}$ & $\begin{array}{c}\text { Seyfert Type } \\
\text { (6) }\end{array}$ & $\begin{array}{l}b / a \\
(7)\end{array}$ & $\begin{array}{l}V_{\text {hel }} \\
(8)\end{array}$ & $\begin{array}{l}D \\
(9)\end{array}$ & $\begin{array}{l}M_{B} \\
(10)\end{array}$ \\
\hline ESO $137-G 34 \ldots \ldots$ & $\mathrm{Mu}$ & DSS $(R)$ & $\mathrm{SAB}(\mathrm{s}) 0 / \mathrm{a} ?$ & 0.0 & Sy2 (Veron) & 0.76 & 2747 & 36.6 & -23.47 \\
\hline IC $2560 \ldots \ldots \ldots \ldots$ & $\mathrm{Mu}$ & $\mathrm{DSS}(R)$ & $\left(\mathrm{R}^{\prime}:\right) \mathrm{SB}(\mathrm{r}) \mathrm{bc}$ & 3.3 & Sy2 (Veron) & 0.63 & 2925 & 39.0 & -21.04 \\
\hline IC $5063 \ldots \ldots \ldots \ldots$ & $\mathrm{Mu}$ & $\operatorname{ESO}(V), \operatorname{DSS}(R)$ & $\mathrm{SA}(\mathrm{s}) 0+:$ & -0.8 & Sy1.9 (Veron) & 0.67 & 3402 & 45.4 & -20.71 \\
\hline Mrk $573 \ldots \ldots \ldots \ldots$ & $\mathrm{Mu}, \mathrm{Po}$ & $\operatorname{DSS}(R)$ & (R)SAB(rs) $0+$ : & -1.0 & Sy2 (Veron) & 1.00 & 5174 & 69.0 & -20.62 \\
\hline Mrk 1066 .......... & $\mathrm{Mu}$ & $\operatorname{DSS}(R)$ & $(\mathrm{R}) \mathrm{SB}(\mathrm{s}) 0+$ & -1.0 & Sy2 (Veron) & 0.59 & 3605 & 48.1 & -20.37 \\
\hline Mrk $1210 \ldots \ldots \ldots$ & $\mathrm{Mu}$ & $\operatorname{DSS}(R)$ & $\mathrm{Sa}$ & 1.0 & Sy1h (Veron) & 1.00 & 4046 & 53.9 & -19.45 \\
\hline NGC $788 \ldots . . . .$. & $\mathrm{Mu}$ & $\operatorname{DSS}(R)$ & $\mathrm{SA}(\mathrm{s}) 0 / \mathrm{a}:$ & 0.0 & Sy1h (Veron) & 0.74 & 4078 & 54.4 & -20.88 \\
\hline NGC 1068 ........ & $\mathrm{Th}$ & $2 \mathrm{M}(H), \operatorname{DSS}(R)$ & (R)SA(rs)b & 3.0 & Sy1h (Veron) & 0.85 & 1137 & 14.4 & -21.32 \\
\hline NGC $1241 \ldots . . . .$. & $\mathrm{Mu}$ & $2 \mathrm{M}(H), \mathrm{DSS}(R)$ & $\mathrm{SB}(\mathrm{rs}) \mathrm{b}$ & 3.0 & Sy2 (Veron) & 0.61 & 4062 & 26.6 & -20.04 \\
\hline NGC 1365 ........ & $\mathrm{St}$ & $\operatorname{ESO}(R), \operatorname{DSS}(R)$ & $\left(\mathrm{R}^{\prime}\right) \mathrm{SBb}(\mathrm{s}) \mathrm{b}$ & 3.0 & Sy1.5 (Veron) & 0.55 & 1636 & 16.9 & -21.21 \\
\hline NGC 1667 ........ & $\mathrm{Mu}$ & $2 \mathrm{M}(H), \operatorname{DSS}(R)$ & $\mathrm{SAB}(\mathrm{r}) \mathrm{c}$ & 5.0 & Sy2 (Veron) & 0.78 & 4547 & 60.6 & -21.50 \\
\hline NGC $1672 \ldots . . . .$. & $\mathrm{Mu}$ & $\operatorname{DSS}(R)$ & $\left(\mathrm{R}^{\prime} \_1:\right) \mathrm{SB}(\mathrm{r}) \mathrm{bc}$ & 3.0 & Sy2 (Veron82) & 0.83 & 1350 & 14.5 & -20.56 \\
\hline NGC $2639 \ldots \ldots \ldots$ & $\mathrm{Mu}$ & $2 \mathrm{M}(H), \operatorname{DSS}(R)$ & (R)SA(r)a:? & 1.0 & Sy1.9 (Veron) & 0.61 & 3336 & 44.5 & -21.05 \\
\hline NGC 2985 ......... & $\mathrm{Mu}, \mathrm{St}$ & $2 \mathrm{M}(H), \operatorname{DSS}(R)$ & (R')SA(rs)ab & 2.0 & Sy1.9(Veron) & 0.79 & 1322 & 22.4 & -20.77 \\
\hline NGC $3031 \ldots . . .$. & St & $2 \mathrm{M}(H), \mathrm{ING}(I), \mathrm{DSS}(R)$ & $\mathrm{SA}(\mathrm{s}) \mathrm{ab}$ & 2.0 & Sy1.5 (Veron) & 0.52 & -34 & 1.4 & -18.34 \\
\hline NGC $3081 \ldots \ldots \ldots$ & $\mathrm{Mu}$ & $2 \mathrm{M}(H), \operatorname{DSS}(R)$ & $\left(\mathrm{R} \_1\right) \mathrm{SAB}(\mathrm{r}) 0 / \mathrm{a}$ & 0.0 & Sy2 (Veron) & 0.78 & 2385 & 32.5 & -19.97 \\
\hline NGC 3227 ......... & $\mathrm{Ri}$ & $2 \mathrm{M}(H), \operatorname{DSS}(R)$ & $\mathrm{SAB}(\mathrm{s})$ pec & 1.0 & Sy1.5 (Veron) & 0.67 & 1157 & 20.6 & -20.39 \\
\hline NGC $3486 \ldots \ldots \ldots$. & $\mathrm{Mu}$ & $2 \mathrm{M}(H), \operatorname{DSS}(R)$ & $\mathrm{SAB}(\mathrm{r}) \mathrm{c}$ & 5.0 & Sy2 (Ho) & 0.74 & 681 & 7.4 & -18.58 \\
\hline NGC 3516 ........ & $\mathrm{Mu}$ & $2 \mathrm{M}(H), \operatorname{DSS}(R)$ & (R)SB(s)ôô: & -2.0 & Sy1.5 (Veron) & 0.78 & 2649 & 38.9 & -20.81 \\
\hline NGC 3718 ........ & $\mathrm{Mu}$ & $2 \mathrm{M}(H), \operatorname{DSS}(R)$ & $\mathrm{SB}(\mathrm{s}) \mathrm{a}$ pec & 1.0 & Sy1 (Veron) & 0.49 & 994 & 17.0 & -19.96 \\
\hline NGC 3786 ......... & Po & $2 \mathrm{M}(H), \operatorname{DSS}(R)$ & $\mathrm{SAB}(\mathrm{rs}) \mathrm{a}$ pec & 1.0 & Sy1.8 (GR) & 0.59 & 2678 & 41.6 & -18.18 \\
\hline NGC 3982 ......... & $\mathrm{Mu}, \mathrm{Po}$ & CF $(H)$, DSS $(R)$ & $\mathrm{SAB}(\mathrm{r}) \mathrm{b}:$ & 3.0 & Sy1.9 (Veron) & 0.87 & 1109 & 17.0 & -19.47 \\
\hline NGC 4117 ........ & $\mathrm{Mu}$ & $2 \mathrm{M}(H), \mathrm{DSS}(R)$ & Sôô: & -2.3 & Sy2 (Veron) & 0.49 & 958 & 17.0 & -17.12 \\
\hline NGC $4151 \ldots \ldots .$. & $\mathrm{Th}$ & $\mathrm{CF}(H), 2 \mathrm{M}(H), \mathrm{DSS}(R)$ & (R')SAB(rs)ab: & 2.0 & Sy1.5 (Veron) & 0.71 & 995 & 20.3 & -20.83 \\
\hline NGC $4253 \ldots \ldots \ldots$ & $\mathrm{Mu}$ & $\operatorname{DSS}(R)$ & (R')SB(s)a: & 1.0 & Sy1.5 (Veron) & 0.80 & 3876 & 56.7 & -19.98 \\
\hline NGC $4303 \ldots . . . .$. & $\mathrm{Mu}$ & $\operatorname{DSS}(R)$ & $\mathrm{SAB}(\mathrm{rs}) \mathrm{bc}$ & 4.0 & Sy2 (Veron) & 0.89 & 1566 & 15.2 & -20.79 \\
\hline NGC 4593 ........ & $\mathrm{Mu}$ & $2 \mathrm{M}(H), \operatorname{DSS}(R)$ & (R)SB(rs)b & 3.0 & Sy1 (Veron) & 0.74 & 2698 & 39.5 & -21.55 \\
\hline NGC 4725 ........ & $\mathrm{Mu}$ & Frei $(I), \operatorname{DSS}(R)$ & SAB(r)ab pec & 2.0 & Sy2 (Ho) & 0.71 & 1206 & 12.4 & -20.69 \\
\hline NGC $4785 \ldots \ldots \ldots$ & $\mathrm{Mu}$ & $\mathrm{Mz}(K), \mathrm{DSS}(R)$ & (R')SAB(r)ab & 3.0 & Sy2 (Veron) & 0.53 & 3735 & 49.8 & -21.70 \\
\hline NGC 4939 ........ & $\mathrm{Mu}$ & Co $(K)$, DSS $(R)$ & $\mathrm{SA}(\mathrm{s}) \mathrm{bc}$ & 4.0 & Sy2 (Veron) & 0.51 & 3111 & 44.3 & -22.03 \\
\hline NGC 4941 ........ & $\mathrm{Mu}$ & $2 \mathrm{M}(H), \operatorname{DSS}(R)$ & (R)SAB(r)ab: & 2.0 & Sy2 (Veron) & 0.62 & 1108 & 6.4 & -17.39 \\
\hline NGC 4968 ........ & $\mathrm{Mu}$ & $\operatorname{DSS}(R)$ & (R')SABÔ̂̂ & -2.0 & Sy2 (Veron) & 0.46 & 2957 & 39.4 & -19.58 \\
\hline NGC $5033 \ldots . . . .$. & $\mathrm{Mu}, \mathrm{Po}$ & $2 \mathrm{M}(H), \operatorname{DSS}(R)$ & $\mathrm{SA}(\mathrm{s}) \mathrm{c}$ & 5.0 & Sy1.9 (Veron) & 0.47 & 875 & 18.7 & -21.15 \\
\hline NGC $5135 \ldots \ldots \ldots$ & $\mathrm{Mu}$ & DSS $(R)$ & $\mathrm{SB}(1) \mathrm{ab}$ & 2.0 & Sy2 (Veron) & 0.69 & 4112 & 54.8 & -21.32 \\
\hline NGC 5194 ........ & $\mathrm{Sc}$ & $\operatorname{ING}(I), 2 \mathrm{M}(H)$ & $\mathrm{SA}(\mathrm{s}) \mathrm{bc}$ pec & 4.0 & Sy2 (Veron) & 0.62 & 463 & 7.7 & -20.76 \\
\hline NGC $5273 \ldots \ldots \ldots$ & Po & $2 \mathrm{M}(H), \operatorname{DSS}(R)$ & SA(s)ôô & -2.0 & Sy1.9 (Veron) & 0.91 & 1089 & 21.3 & -19.26 \\
\hline NGC $5283 \ldots \ldots \ldots$ & Po & $\operatorname{DSS}(R)$ & So? & -2.0 & Sy2 (Veron) & 0.91 & 2700 & 41.4 & -18.97 \\
\hline NGC 5347 ........ & $\mathrm{Mu}$ & $2 \mathrm{M}(H), \mathrm{DSS}(R)$ & $\left(\mathrm{R}^{\prime}\right) \mathrm{SB}(\mathrm{rs}) \mathrm{ab}$ & 2.0 & Sy2 (Veron) & 0.79 & 2335 & 36.7 & -19.72 \\
\hline NGC 5427 ........ & $\mathrm{Mu}$ & $\mathrm{Ju}(H)$ & $\mathrm{SA}(\mathrm{s}) \mathrm{c}$ pec & 5.0 & Sy2 (Veron) & 0.85 & 2618 & 38.1 & -21.17 \\
\hline NGC 5548 ........ & $\mathrm{Ri}$ & $\mathrm{CF}(H), \operatorname{DSS}(R)$ & $\left(\mathrm{R}^{\prime}\right) \mathrm{SA}(\mathrm{s}) 0 / \mathrm{a}$ & 0.0 & Sy1.5 (Veron) & 0.93 & 5149 & 68.7 & -21.37 \\
\hline NGC $5643 \ldots \ldots \ldots$ & $\mathrm{Mu}$ & $\operatorname{DSS}(R)$ & $\mathrm{SAB}(\mathrm{rs}) \mathrm{c}$ & 5.0 & Sy2 (Veron) & 0.87 & 1199 & 16.9 & -20.91 \\
\hline NGC $5695 \ldots . . .$. & Po & $2 \mathrm{M}(H), \operatorname{DSS}(R)$ & $\mathrm{SBb}$ & 3.0 & Sy2 (Veron) & 0.73 & 4225 & 56.3 & -20.38 \\
\hline NGC 5929 ........ & $\mathrm{Mu}, \mathrm{Po}$ & $2 \mathrm{M}(H), \operatorname{DSS}(R)$ & Sab: pec & 2.0 & Sy2 (Huchra) & 0.93 & 2492 & 38.5 & -19.93 \\
\hline NGC 5953 ........ & $\mathrm{Mu}$ & $\operatorname{DSS}(R)$ & SAa: pec & 1.0 & Sy2 (Veron) & 0.83 & 1965 & 33.0 & -19.29 \\
\hline NGC $6221 \ldots \ldots \ldots$ & $\mathrm{Mu}$ & $\operatorname{DSS}(R)$ & $\mathrm{SB}(\mathrm{s}) \mathrm{bc}$ pec & 5.0 & Sy1 (Heckman) & 0.69 & 1482 & 19.4 & -21.67 \\
\hline NGC $6300 \ldots \ldots . .$. & $\mathrm{Mu}$ & $\operatorname{ESO}(R)$ & $\mathrm{SB}(\mathrm{rs}) \mathrm{b}$ & 3.0 & Sy2 (Veron) & 0.66 & 1110 & 14.3 & -20.58 \\
\hline NGC 6814 ........ & $\mathrm{Mu}$ & $\mathrm{CF}(H)$ & $\mathrm{SAB}(\mathrm{rs}) \mathrm{bc}$ & 4.0 & Sy1.5 (Veron) & 0.93 & 1563 & 22.8 & -20.47 \\
\hline NGC $6890 \ldots \ldots \ldots$ & $\mathrm{Mu}$ & $\operatorname{DSS}(R)$ & (R')SA(r:)ab & 3.0 & Sy1.9 (Veron) & 0.79 & 2419 & 31.8 & -19.69 \\
\hline NGC $6951 \ldots \ldots .$. & $\mathrm{Mu}$ & ING $(I), \operatorname{DSS}(R)$ & $\mathrm{SAB}(\mathrm{rs}) \mathrm{bc}$ & 4.0 & Sy2 (Veron) & 0.83 & 1424 & 24.1 & -21.20 \\
\hline NGC $7130 \ldots \ldots . .$. & $\mathrm{Mu}$ & $2 \mathrm{M}(H), \operatorname{DSS}(R)$ & Sa pec & 1.0 & Sy1.9 (Veron) & 0.93 & 4842 & 64.6 & -21.17 \\
\hline NGC 7469 ........ & Sc & $\mathrm{CF}(H), \operatorname{DSS}(R)$ & (R')SAB(rs)a & 1.0 & Sy1.5 (Veron) & 0.73 & 4892 & 65.2 & -21.43 \\
\hline NGC 7479 ........ & $\mathrm{St}$ & $2 \mathrm{M}(H), \operatorname{DSS}(R)$ & $\mathrm{SB}(\mathrm{s}) \mathrm{c}$ & 5.0 & Sy1.9 (Veron) & 0.76 & 2381 & 32.4 & -21.33 \\
\hline NGC $7496 \ldots \ldots \ldots$ & $\mathrm{Mu}$ & $\operatorname{DSS}(R)$ & $\left(\mathrm{R}^{\prime}:\right) \mathrm{SB}(\mathrm{rs}) \mathrm{bc}$ & 3.0 & Sy2 (Veron) & 0.91 & 1649 & 20.1 & -19.68 \\
\hline NGC $7682 \ldots \ldots \ldots$ & Po & $\mathrm{CF}(H), \mathrm{DSS}(R)$ & $\mathrm{SB}(\mathrm{r}) \mathrm{ab}$ & 2.0 & Sy2 (Veron) & 0.92 & 5134 & 68.5 & -20.51 \\
\hline NGC 7743 ........ & $\mathrm{Mu}$ & $\operatorname{ING}(I), \operatorname{DSS}(R)$ & $(\mathrm{R}) \mathrm{SB}(\mathrm{s}) 0+$ & -1.0 & Sy2 (Veron) & 0.85 & 1710 & 24.4 & -19.78 \\
\hline UGC $1395 \ldots \ldots \ldots$ & Po & $\operatorname{DSS}(R)$ & $\mathrm{SA}(\mathrm{rs}) \mathrm{b}$ & 3.0 & Sy1.9 (Veron) & 0.77 & 5208 & 69.4 & -20.35 \\
\hline
\end{tabular}

Note.-Col. (1): Galaxy names. Col. (2): Source of HST data: $\mathrm{Mu}=$ Mulchaey; $\mathrm{St}=$ Stiavelli; $\mathrm{Po}=\mathrm{Pogge} ; \mathrm{Th}=\mathrm{Thompson} ; \mathrm{Ri}=\mathrm{Rieke}$; $\mathrm{Sc}=\mathrm{Scoville}$ Col. (3): Source of outer galaxy data: $2 \mathrm{M}=2 \mathrm{MASS}$ Sky Survey; DSS = Digital Sky Survey; ING = ING data archive; ESO = ESO data archive; $\mathrm{CF}=$ Peletier et al. 1999a; Frei $=$ Frei 1999; Mz = Márquez et al. 1999; Co = F. Combes et al. 2002, in preparation; Ju = Jungwiert et al. 1997; band is included in parentheses. Col. (4): Morphological type (from NED). Col. (5): Numerical morphology " T" type (from RC3). Col. (6): Seyfert type and reference: Veron $=$ Veron-Cetty \& Veron 1991; Veron82 = Veron, Veron, \& Zuiderwijk 1981; Heckman = T. Heckman 2001, private communication; Ho = Ho, Filippenko, \& Sargent 1997b; GR = Goodrich \& Osterbrock 1983; Huchra = Huchra, Wyatt, \& Davis 1982. Col. (7): Axial ratio (minor axis/major axis) from NED. Col. (8): Heliocentric velocity in $\mathrm{km} \mathrm{s}^{-1}$ (from NED). Col. (9): Distance in Mpc. Col. (10): Absolute $B$ magnitude, calculated from $B_{T, 0}$, which was taken from RC3, and the distance in col. (9). Table 4 is also available in machine-readable form in the electronic edition of the Astrophysical Journal. 
TABLE 5

Properties of Non-Seyfert Control Galaxy SAmple

\begin{tabular}{|c|c|c|c|c|c|c|c|c|}
\hline $\begin{array}{l}\text { Galaxy } \\
\text { (1) }\end{array}$ & $\begin{array}{c}H S T \text { Source } \\
\text { (2) }\end{array}$ & $\begin{array}{l}\text { Other Source } \\
\text { (3) }\end{array}$ & $\begin{array}{c}\text { Classification } \\
\text { (4) }\end{array}$ & $\begin{array}{c}\mathrm{T} \\
(5)\end{array}$ & $\begin{array}{l}b / a \\
(6)\end{array}$ & $\begin{array}{l}V_{\text {hel }} \\
\text { (7) }\end{array}$ & $\begin{array}{l}D \\
(8)\end{array}$ & $\begin{array}{l}M_{B} \\
(9)\end{array}$ \\
\hline IC $5267 \ldots \ldots \ldots$ & $\mathrm{Mu}$ & $\operatorname{DSS}(R)$ & (R)SA(rs)0/a & 0.0 & 0.74 & 1713 & 21.0 & -20.32 \\
\hline NGC $214 \ldots . .$. & $\mathrm{Mu}$ & $\operatorname{DSS}(R)$ & $\mathrm{SAB}(\mathrm{r}) \mathrm{c}$ & 5.0 & 0.74 & 4534 & 60.5 & -21.28 \\
\hline NGC $289 \ldots \ldots$. & St & $2 \mathrm{M}(H), \operatorname{DSS}(R)$ & $\mathrm{SAB}(\mathrm{rs}) \mathrm{bc}$ & 4.0 & 0.71 & 1628 & 19.4 & -20.04 \\
\hline NGC $357 \ldots \ldots$. & $\mathrm{Mu}$ & $2 \mathrm{M}(H), \mathrm{DSS}(R)$ & $\mathrm{SB}(\mathrm{r}) 0 / \mathrm{a}:$ & 0.0 & 0.72 & 2406 & 32.1 & -19.93 \\
\hline NGC $404 \ldots . .$. & $\mathrm{Mu}$ & $2 \mathrm{M}(H), \operatorname{DSS}(R)$ & $\mathrm{SA}(\mathrm{s}) 0-:$ & -3.0 & 1.00 & -48 & 2.4 & -15.98 \\
\hline NGC $488 \ldots \ldots$. & $\mathrm{St}$ & ING $(I), \operatorname{DSS}(R)$ & $\mathrm{SA}(\mathrm{r}) \mathrm{b}$ & 3.0 & 0.74 & 2272 & 29.3 & -21.43 \\
\hline NGC $628 \ldots . .$. & $\mathrm{Mu}$ & $\operatorname{DSS}(R)$ & $\mathrm{SA}(\mathrm{s}) \mathrm{c}$ & 5.0 & 0.90 & 657 & 9.7 & -20.17 \\
\hline NGC $772 \ldots \ldots$. & $\mathrm{St}$ & $2 \mathrm{M}(H), \operatorname{DSS}(R)$ & $\mathrm{SA}(\mathrm{s}) \mathrm{b}$ & 3.0 & 0.59 & 2472 & 32.6 & -22.02 \\
\hline NGC $864 \ldots . . .$. & $\mathrm{Mu}$ & $\operatorname{ING}(R), \operatorname{DSS}(R)$ & $\mathrm{SAB}(\mathrm{rs}) \mathrm{c}$ & 5.0 & 0.76 & 1562 & 20.0 & -20.25 \\
\hline NGC $1345 \ldots . .$. & St & $\operatorname{DSS}(R)$ & SB(s)c pec: & 4.5 & 0.74 & 1529 & 18.1 & -17.49 \\
\hline NGC $1398 \ldots . .$. & $\mathrm{Mu}, \mathrm{St}$ & $2 \mathrm{M}(H), \operatorname{DSS}(R)$ & (R_1R'_2)SB(rs)ab & 2.0 & 0.76 & 1407 & 16.1 & -20.64 \\
\hline NGC $1530 \ldots . .$. & $\mathrm{Mu}$ & $2 \mathrm{M}(H), \operatorname{DSS}(R)$ & $\mathrm{SB}(\mathrm{rs}) \mathrm{b}$ & 3.0 & 0.52 & 2461 & 36.6 & -21.40 \\
\hline NGC $1638 \ldots \ldots$ & $\mathrm{Mu}$ & $2 \mathrm{M}(H), \operatorname{DSS}(R)$ & $\mathrm{SAB}(\mathrm{rs}) 0 \hat{0} 0 \hat{?}$ & -2.3 & 0.75 & 3320 & 44.3 & -20.46 \\
\hline NGC $1961 \ldots . .$. & $\mathrm{Mu}$ & ING $(R)$, DSS $(R)$ & $\mathrm{SAB}(\mathrm{rs}) \mathrm{c}$ & 5.0 & 0.65 & 3934 & 52.5 & -22.59 \\
\hline NGC $2179 \ldots . .$. & $\mathrm{Mu}$ & $2 \mathrm{M}(H), \operatorname{DSS}(R)$ & $(\mathrm{R}) \mathrm{SA}(\mathrm{r}) \hat{0}+?$ & 0.0 & 0.69 & 2885 & 34.2 & -19.84 \\
\hline NGC $2196 \ldots . .$. & $\mathrm{St}$ & $2 \mathrm{M}(H), \operatorname{DSS}(R)$ & $\left(\mathrm{R}^{\prime}:\right) \mathrm{SA}(\mathrm{rs}) \mathrm{ab}$ & 1.0 & 0.78 & 2321 & 28.8 & -20.92 \\
\hline NGC $2223 \ldots . .$. & $\mathrm{Mu}$ & $2 \mathrm{M}(H), \operatorname{DSS}(R)$ & $\mathrm{SB}(\mathrm{rs}) \mathrm{bc}$ & 3.0 & 0.85 & 2722 & 33.7 & -20.78 \\
\hline NGC $2276 \ldots . .$. & $\mathrm{Mu}$ & $\operatorname{ING}(I), \operatorname{DSS}(R)$ & $\mathrm{SAB}(\mathrm{rs}) \mathrm{c}$ & 5.0 & 0.95 & 2410 & 36.8 & -21.08 \\
\hline NGC $2339 \ldots . .$. & $\mathrm{St}$ & $\operatorname{DSS}(R)$ & $\mathrm{SAB}(\mathrm{rs}) \mathrm{bc}$ & 4.0 & 0.76 & 2206 & 30.9 & -20.97 \\
\hline NGC $2344 \ldots \ldots$. & St & $\operatorname{DSS}(R)$ & $\mathrm{SA}(\mathrm{rs}) \mathrm{c}:$ & 4.5 & 0.98 & 974 & 16.0 & -18.54 \\
\hline NGC $2460 \ldots . .$. & St & ING $(I), \operatorname{DSS}(R)$ & $\mathrm{SA}(\mathrm{s}) \mathrm{a}$ & 1.0 & 0.76 & 1442 & 23.6 & -19.57 \\
\hline NGC $2566 \ldots . .$. & $\mathrm{St}$ & $2 \mathrm{M}(H), \mathrm{DSS}(R)$ & $\left(\mathrm{R}^{\prime}\right) \mathrm{SB}(\mathrm{r}) \mathrm{ab}$ & 2.5 & 0.68 & 1637 & 21.1 & -21.52 \\
\hline NGC $3032 \ldots . .$. & $\mathrm{Mu}$ & $\operatorname{DSS}(R)$ & SAB(r)ố & -2.0 & 0.89 & 1533 & 24.5 & -19.11 \\
\hline NGC $3169 \ldots \ldots$ & $\mathrm{St}$ & ING $(I)$, DSS $(R)$ & SA(s)a pec & 1.0 & 0.63 & 1233 & 19.7 & -20.51 \\
\hline NGC $3277 \ldots \ldots$ & $\mathrm{St}$ & $2 \mathrm{M}(H), \operatorname{DSS}(R)$ & $\mathrm{SA}(\mathrm{r}) \mathrm{ab}$ & 2.0 & 0.89 & 1408 & 25.0 & -19.62 \\
\hline NGC $3300 \ldots . .$. & $\mathrm{Mu}$ & $2 \mathrm{M}(H), \mathrm{DSS}(R)$ & SAB(r)0̂0̂:? & -2.0 & 0.53 & 3045 & 42.9 & -20.19 \\
\hline NGC $3368 \ldots \ldots$ & $\mathrm{Mu}$ & ING $(I), \operatorname{DSS}(R)$ & $\mathrm{SAB}(\mathrm{rs}) \mathrm{ab}$ & 2.0 & 0.69 & 897 & 8.1 & -19.74 \\
\hline NGC $3865 \ldots \ldots$ & $\mathrm{Mu}$ & $2 \mathrm{M}(H), \mathrm{DSS}(R)$ & SAB(rs)b pec: & 3.0 & 0.75 & 5702 & 76.0 & -21.84 \\
\hline NGC $3928 \ldots . .$. & $\mathrm{St}$ & $\operatorname{DSS}(R)$ & $\mathrm{SA}(\mathrm{s}) \mathrm{b} ?$ & 3.0 & 1.00 & 988 & 17.0 & -18.07 \\
\hline NGC $4030 \ldots .$. & $\mathrm{Mu}$ & Frei $(R), 2 \mathrm{M}(H), \operatorname{DSS}(R)$ & $\mathrm{SA}(\mathrm{s}) \mathrm{bc}$ & 4.0 & 0.72 & 1460 & 25.9 & -20.90 \\
\hline NGC $4143 \ldots . .$. & $\mathrm{Mu}$ & $2 \mathrm{M}(H), \operatorname{DSS}(R)$ & SAB(s)ô̂̂ & -2.0 & 0.61 & 985 & 17.0 & -19.25 \\
\hline NGC $4254 \ldots \ldots$ & $\mathrm{Mu}$ & $2 \mathrm{M}(H), \mathrm{DSS}(R)$ & $\mathrm{SA}(\mathrm{s}) \mathrm{c}$ & 5.0 & 0.87 & 2406 & 16.8 & -21.03 \\
\hline NGC $4260 \ldots . .$. & $\mathrm{Mu}$ & $\operatorname{DSS}(R)$ & $\mathrm{SB}(\mathrm{s}) \mathrm{a}$ & 1.0 & 0.50 & 1958 & 35.1 & -20.42 \\
\hline NGC $4384 \ldots \ldots$. & $\mathrm{St}$ & $\operatorname{DSS}(R)$ & $\mathrm{Sa}$ & 1.0 & 0.77 & 2513 & 36.6 & -19.44 \\
\hline NGC $4569 \ldots \ldots$ & St & $2 \mathrm{M}(H), \operatorname{DSS}(R)$ & $\mathrm{SAB}(\mathrm{rs}) \mathrm{ab}$ & 2.0 & 0.46 & -235 & 16.8 & -21.34 \\
\hline NGC $4750 \ldots \ldots$ & St & $2 \mathrm{M}(H), \mathrm{DSS}(R)$ & (R)SA(rs)ab & 2.0 & 0.91 & 1623 & 26.1 & -20.12 \\
\hline NGC $5054 \ldots \ldots$ & $\mathrm{Mu}$ & $2 \mathrm{M}(H), \operatorname{DSS}(R)$ & $\mathrm{SA}(\mathrm{s}) \mathrm{bc}$ & 4.0 & 0.59 & 1741 & 27.3 & -21.05 \\
\hline NGC $5064 \ldots . .$. & $\mathrm{Mu}$ & $\operatorname{DSS}(R)$ & $\left(\mathrm{R}^{\prime}:\right) \mathrm{SA}(\mathrm{s}) \mathrm{ab}$ & 2.5 & 0.46 & 2980 & 39.5 & -21.31 \\
\hline NGC $5326 \ldots \ldots$ & $\mathrm{Pe}$ & $2 \mathrm{M}(H), \operatorname{DSS}(R)$ & SAa: & 1.0 & 0.50 & 2520 & 37.8 & -20.31 \\
\hline NGC $5377 \ldots \ldots$ & $\mathrm{St}$ & $2 \mathrm{M}(H), \mathrm{DSS}(R)$ & $(\mathrm{R}) \mathrm{SB}(\mathrm{s}) \mathrm{a}$ & 1.0 & 0.56 & 1793 & 31.0 & -20.52 \\
\hline NGC $5383 \ldots . .$. & $\mathrm{Mu}$ & $\mathrm{Sh}(K), 2 \mathrm{M}(H), \mathrm{DSS}(R)$ & $\left(\mathrm{R}^{\prime}\right) \mathrm{SB}(\mathrm{rs}) \mathrm{b}: \mathrm{pec}$ & 3.0 & 0.85 & 2550 & 37.8 & -20.94 \\
\hline NGC $5448 \ldots \ldots$ & $\mathrm{St}$ & $\operatorname{ING}(I), \operatorname{DSS}(R)$ & (R)SAB(r)a & 1.0 & 0.45 & 2028 & 32.6 & -20.85 \\
\hline NGC $5614 \ldots \ldots$ & $\mathrm{Mu}$ & $\operatorname{DSS}(R)$ & $\mathrm{SA}(\mathrm{r}) \mathrm{ab}$ pec & 2.0 & 0.80 & 3892 & 51.9 & -21.21 \\
\hline NGC $5678 \ldots . .$. & $\mathrm{St}$ & $2 \mathrm{M}(H), \operatorname{DSS}(R)$ & $\mathrm{SAB}(\mathrm{rs}) \mathrm{b}$ & 3.0 & 0.49 & 1922 & 35.6 & -21.09 \\
\hline NGC $5739 \ldots . .$. & $\mathrm{Mu}$ & $\operatorname{DSS}(R)$ & $\mathrm{SAB}(\mathrm{r}) 0+:$ & -0.5 & 0.91 & 5377 & 71.7 & -21.32 \\
\hline NGC $5970 \ldots . .$. & $\mathrm{Mu}$ & ING $(R)$, DSS $(R)$ & $\mathrm{SB}(\mathrm{r}) \mathrm{c}$ & 5.0 & 0.68 & 1957 & 31.6 & -20.66 \\
\hline NGC $5985 \ldots . .$. & $\mathrm{St}$ & $\operatorname{DSS}(R)$ & $\mathrm{SAB}(\mathrm{r}) \mathrm{b}$ & 3.0 & 0.54 & 2517 & 39.2 & -21.59 \\
\hline NGC $6217 \ldots \ldots$. & St & $2 \mathrm{M}(H), \mathrm{DSS}(R)$ & $(\mathrm{R}) \mathrm{SB}(\mathrm{rs}) \mathrm{bc}$ & 4.0 & 0.83 & 1362 & 23.9 & -20.23 \\
\hline NGC $6340 \ldots \ldots$ & $\mathrm{St}$ & $\operatorname{DSS}(R)$ & $\mathrm{SA}(\mathrm{s}) 0 / \mathrm{a}$ & 0.0 & 0.91 & 1198 & 22.0 & -20.04 \\
\hline NGC $7096 \ldots . .$. & $\mathrm{Mu}$ & $\operatorname{DSS}(R)$ & (R')SA(rs)ab & 1.0 & 0.84 & 3100 & 36.7 & -20.18 \\
\hline NGC $7217 \ldots \ldots$ & $\mathrm{St}$ & $2 \mathrm{M}(H), \operatorname{DSS}(R)$ & (R)SA(r)ab & 2.0 & 0.83 & 952 & 16.0 & -20.49 \\
\hline NGC $7280 \ldots \ldots$ & St & $\operatorname{DSS}(R)$ & $\mathrm{SAB}(\mathrm{r}) 0+$ & -1.0 & 0.69 & 1844 & 26.2 & -19.25 \\
\hline NGC $7392 \ldots . .$. & $\mathrm{Mu}$ & $2 \mathrm{M}(H), \operatorname{DSS}(R)$ & $\left(\mathrm{R}^{\prime}:\right) \mathrm{SB}(\mathrm{rs}) \mathrm{ab}$ & 4.0 & 0.62 & 3192 & 42.6 & -20.90 \\
\hline NGC $7421 \ldots \ldots$ & $\mathrm{St}$ & $\operatorname{DSS}(R)$ & $\mathrm{SB}(\mathrm{r}) \mathrm{bc}$ & 4.0 & 0.89 & 1832 & 24.3 & -19.48 \\
\hline NGC $7716 \ldots . .$. & $\mathrm{Mu}$ & ING $(I), \operatorname{DSS}(R)$ & $\mathrm{SAB}(\mathrm{r}) \mathrm{b}:$ & 3.0 & 0.83 & 2571 & 33.7 & -20.13 \\
\hline NGC $7742 \ldots \ldots$ & $\mathrm{St}$ & ING $(I)$, DSS $(R)$ & $\mathrm{SA}(\mathrm{r}) \mathrm{b}$ & 3.0 & 1.00 & 1663 & 22.2 & -19.46 \\
\hline
\end{tabular}

Note.-Col. (1): Galaxy names. Col. (2): Source of HST data: $\mathrm{Mu}=$ Mulchaey; $\mathrm{St}=$ Stiavelli; Pe = Peletier. Col. (3): Source of outer galaxy data: $2 \mathrm{M}=$ 2MASS Sky Survey; DSS = Digital Sky Survey; ING = ING data archive; Frei = Frei 1999; Sh = Sheth et al. 2000; band is included in parentheses. Col. (4): Morphological type (from NED). Col. (5): Numerical morphology "T" type (from RC3). Col. (6): Axial ratio (minor axis/major axis) from NED. Col. (7): Heliocentric velocity in $\mathrm{km} \mathrm{s}^{-1}$ (from NED). Col. (8): Distance in Mpc. Col. (9): Absolute $B$ magnitude, calculated from $B_{T, 0}$, which was taken from RC3, and the distance in col. (8). Table 5 is also available in machinereadable form in the electronic edition of the Astrophysical Journal. 

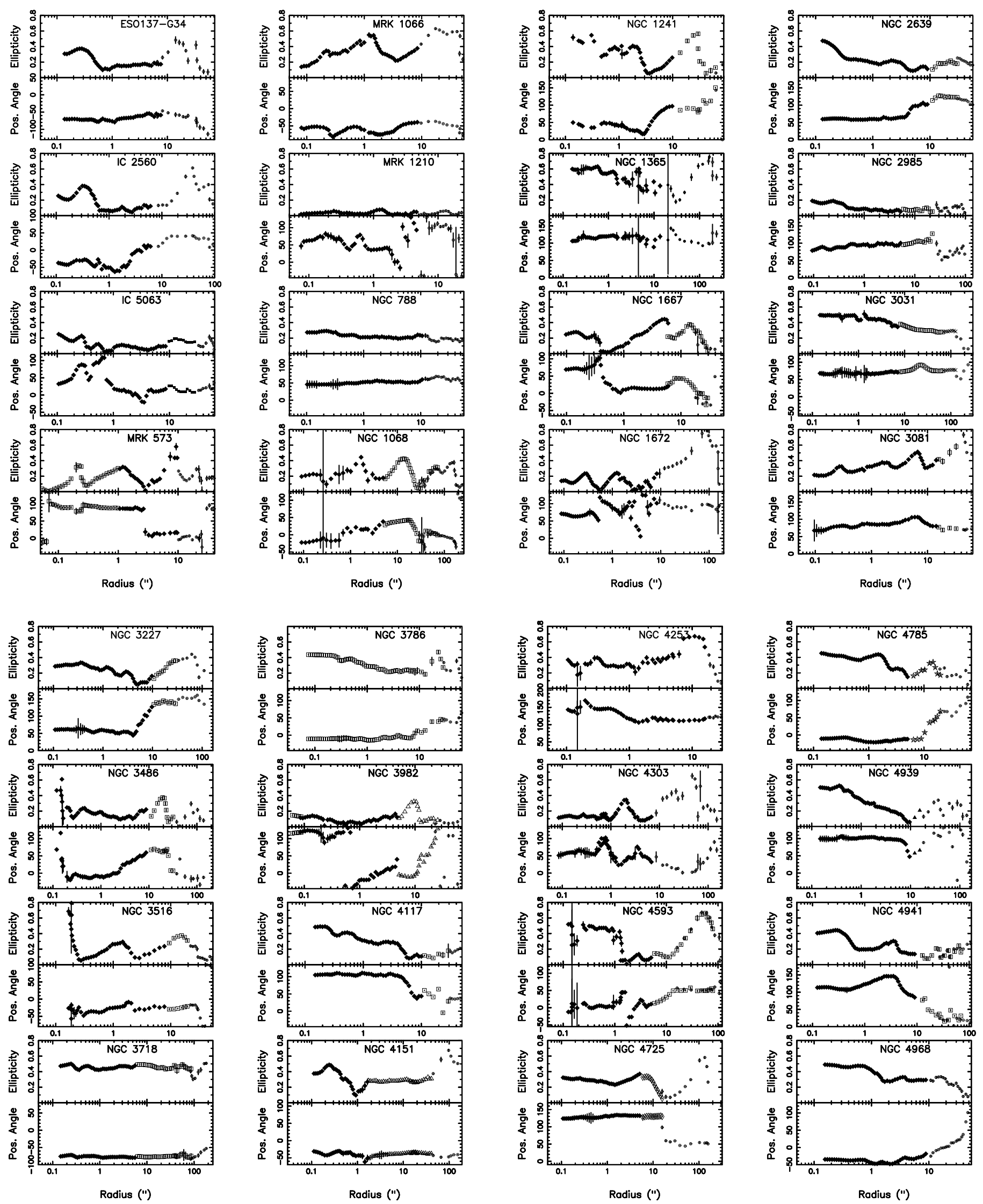

FIG. 10.-Fitted ellipticity and position angle profiles after deprojection for all 56 Seyfert galaxies in our sample. The position angle here does not necessarily have its zero point in the north direction because after deprojection such directional distinctions may not be valid anymore. The uncertainty bars are also shown but are often so small that they cannot be distinguished. The HST data from NICMOS camera 2 ( 0 ".075 pixels) are shown with filled diamonds, NICMOS camera 1 (0.043 pixels) data are shown with big squares, 2MASS data with big open squares, DSS data with small open circles, and the rest of the ground-based data with other symbols. 

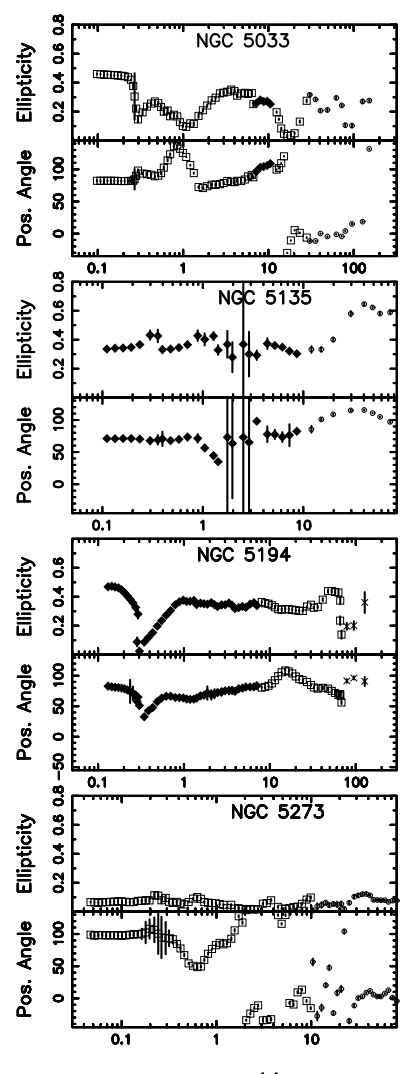

Radius (")
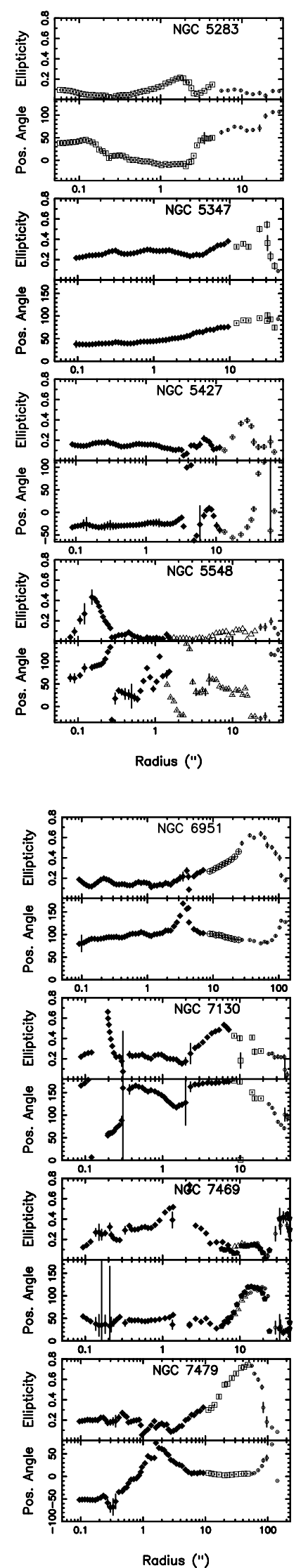
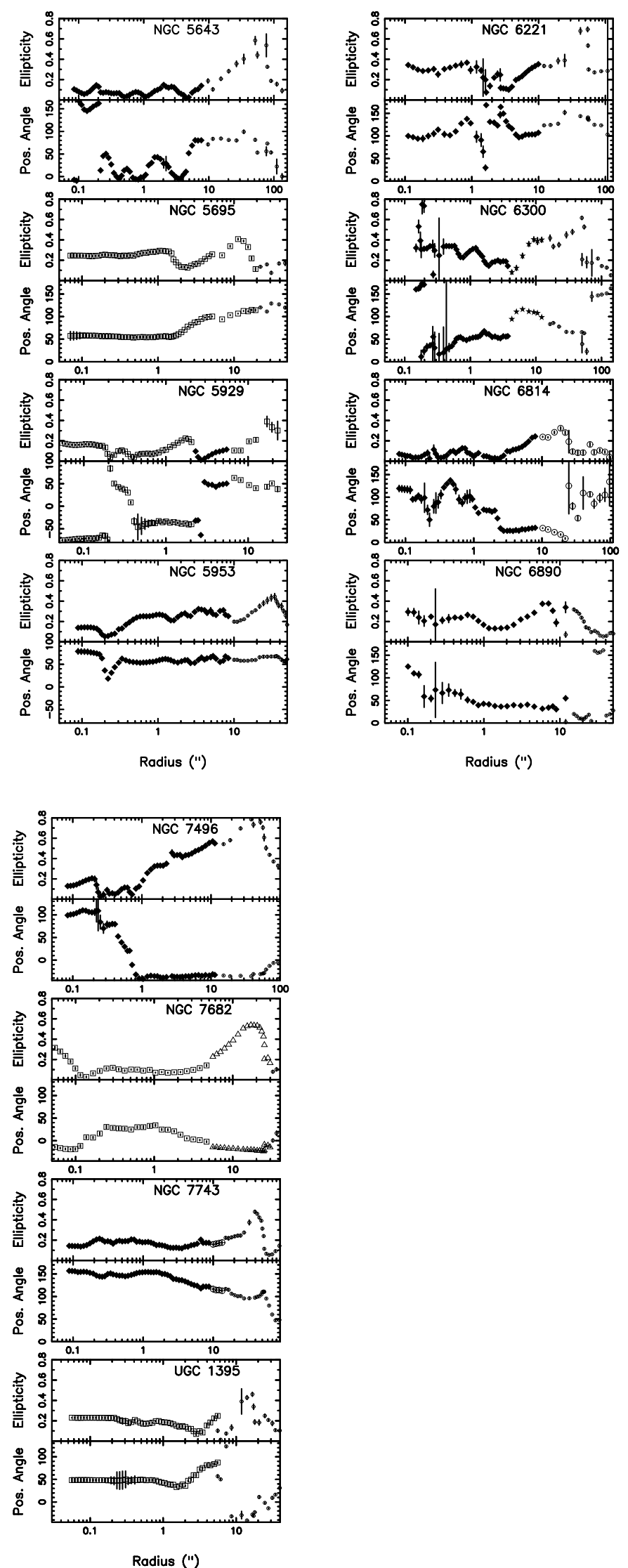

FIG. 10.-Continued 

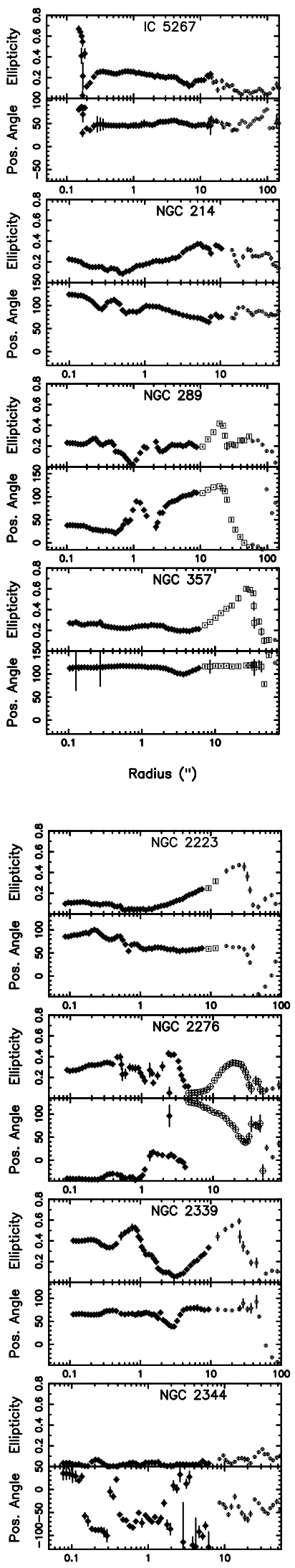

Radius (")
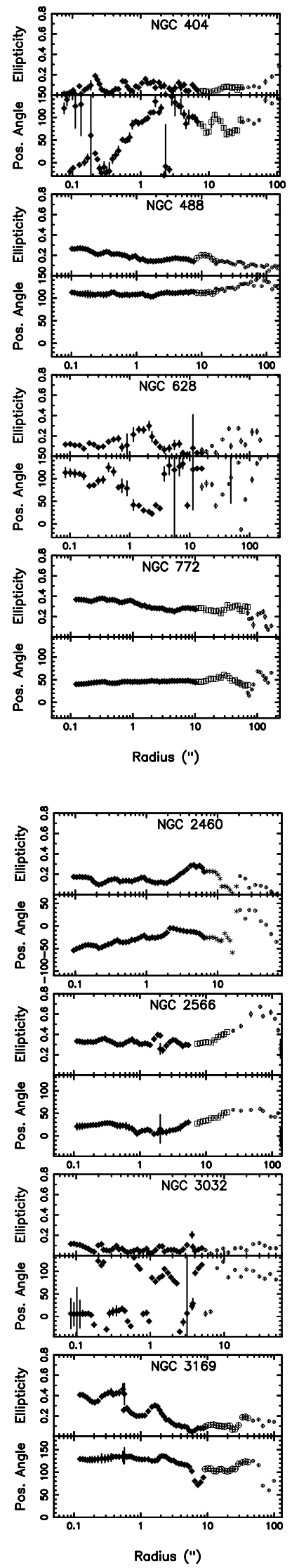
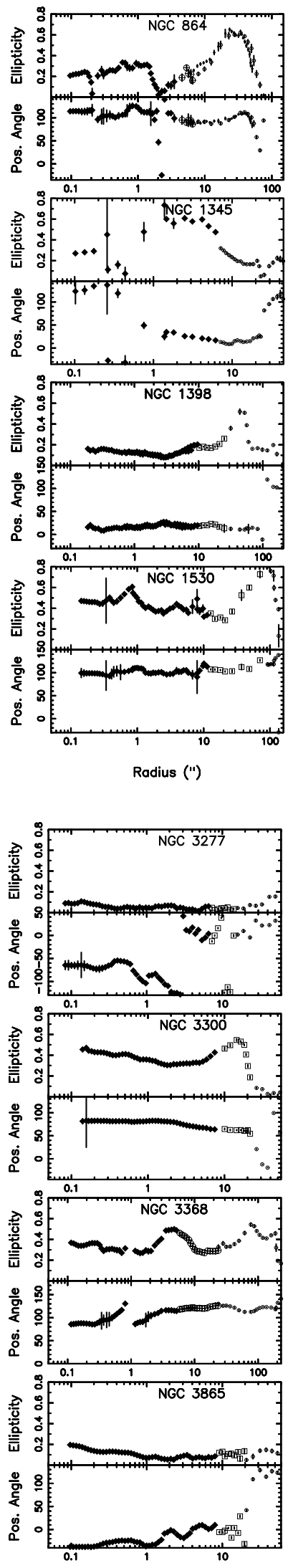

Radius (")
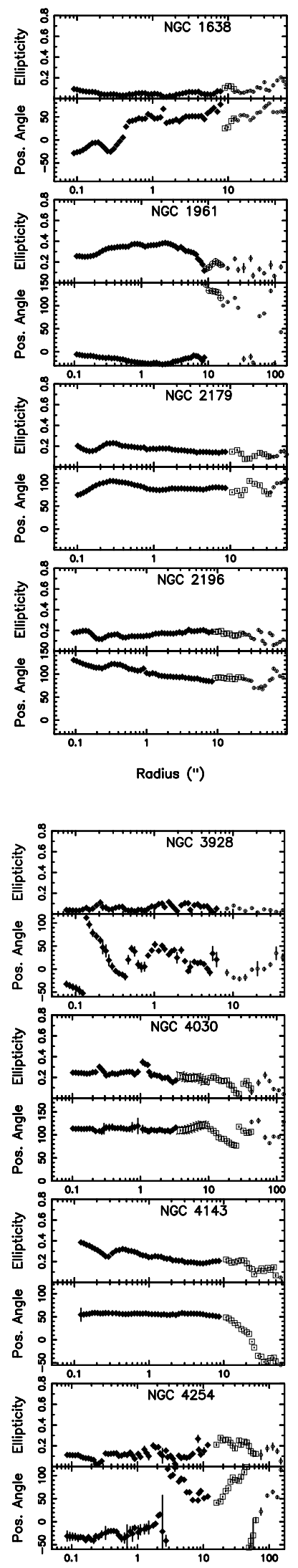

Radius (")

Fig. 11.-Same as Fig. 10, but now for all 56 non-Seyfert galaxies in our sample 

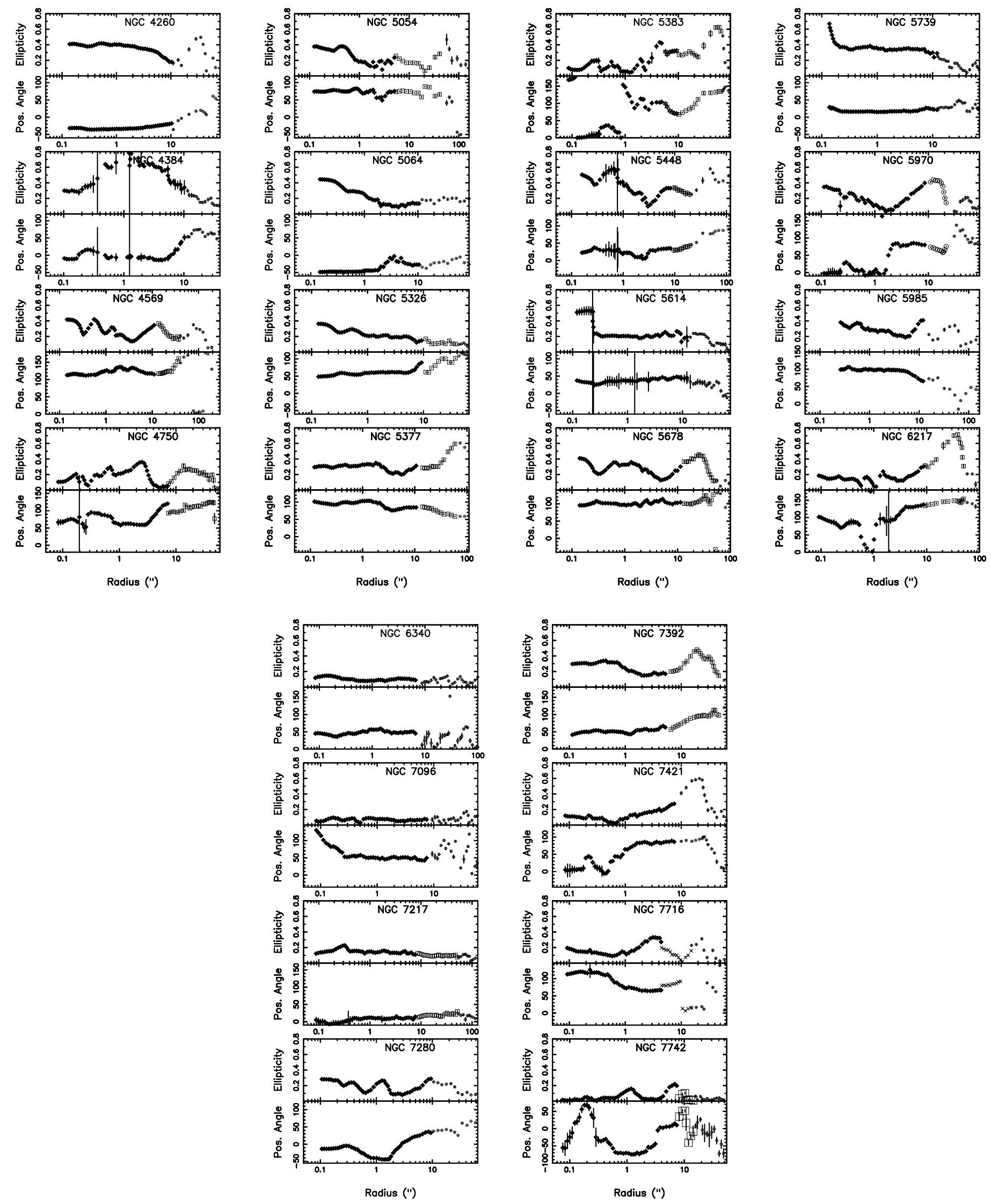

FIG. 11.-Continued 
TABLE 6

Bar Parameters of Seyfert Galaxy SAmple

\begin{tabular}{|c|c|c|c|c|c|}
\hline $\begin{array}{l}\text { Galaxy } \\
\text { (1) }\end{array}$ & $\begin{array}{c}1 \mathrm{kpc} \\
(\operatorname{arcsec}) \\
(2)\end{array}$ & $\begin{array}{l}\text { Deprojected Bar Radii } \\
\text { (arcsec) } \\
(3)\end{array}$ & $\begin{array}{l}\text { Deprojected Bar Radii } \\
\text { (pc) } \\
(4)\end{array}$ & $\begin{array}{l}\text { Deprojected Bar Ellipticities } \\
\text { (5) }\end{array}$ & $\begin{array}{l}\text { Galaxy Diameter } \\
(\operatorname{arcsec}) \\
(6)\end{array}$ \\
\hline ESO $137-G 34 \ldots . .$. & 5.6 & 14 & 2500 & 0.48 & 154 \\
\hline IC $2560 \ldots \ldots \ldots \ldots$ & 5.3 & 38 & 7200 & 0.61 & 203 \\
\hline IC $5063 \ldots \ldots \ldots \ldots$ & 4.5 & $\ldots$ & $\ldots$ & $\ldots$ & 128 \\
\hline Mrk $573 \ldots . . . \ldots \ldots$ & 3.0 & $1.2,9.3,22$ & $400,3100,7300$ & $0.32,0.58,0.29$ & 83 \\
\hline Mrk 1066 ......... & 4.3 & $1.2,17$ & 280,4000 & $0.55,0.63$ & 109 \\
\hline Mrk $1210 \ldots \ldots \ldots$ & 3.8 & $\ldots$ & $\ldots$ & $\ldots$ & 50 \\
\hline NGC $788 \ldots \ldots \ldots$ & 3.8 & $\ldots$ & $\ldots$ & $\ldots$ & 114 \\
\hline NGC $1068 \ldots \ldots \ldots$ & 14.3 & $1.7,14$ & 120,1000 & $0.44,0.42$ & 425 \\
\hline NGC $1241 \ldots \ldots . .$. & 7.8 & $1.8,30$ & 230,3800 & $0.41,0.56$ & 177 \\
\hline NGC 1365 ........ & 12.2 & $4.7,165$ & 390,13500 & $0.60,0.76$ & 673 \\
\hline NGC 1667 ......... & 3.4 & $5.2,14$ & 1400,3800 & $0.45,0.38$ & 112 \\
\hline NGC $1672 \ldots . . . .$. & 14.2 & 92 & 6500 & 0.78 & 396 \\
\hline NGC $2639 \ldots . . . .$. & 4.6 & $\ldots$ & $\ldots$ & $\ldots$ & 112 \\
\hline NGC $2985 \ldots . . . \ldots$ & 9.2 & $\ldots$ & $\ldots$ & $\ldots$ & 274 \\
\hline NGC $3031 \ldots \ldots \ldots$ & 147 & $\ldots$ & $\ldots$ & $\ldots$ & 1653 \\
\hline NGC $3081 \ldots . . . .$. & 6.3 & $6.7,44$ & 1100,7000 & $0.51,0.73$ & 128 \\
\hline NGC 3227 ......... & 10.0 & 62 & 6200 & 0.44 & 330 \\
\hline NGC 3486 ......... & 27.9 & 19 & 680 & 0.38 & 425 \\
\hline NGC 3516 ........ & 5.3 & $1.5,15$ & 280,2800 & $0.29,0.38$ & 104 \\
\hline NGC $3718 \ldots \ldots \ldots$ & 12.1 & $\ldots$ & $\ldots$ & $\ldots$ & 488 \\
\hline NGC 3786 ........ & 5.0 & 23 & 4600 & 0.47 & 131 \\
\hline NGC 3982 ........ & 12.1 & 9.5 & 790 & 0.33 & 141 \\
\hline NGC $4117 \ldots \ldots \ldots$ & 12.1 & $\ldots$ & $\ldots$ & $\ldots$ & 97 \\
\hline NGC $4151 \ldots \ldots \ldots$ & 10.2 & 97 & 9500 & 0.68 & 379 \\
\hline NGC $4253 \ldots \ldots \ldots$ & 3.6 & 11 & 3100 & 0.67 & 59 \\
\hline NGC $4303 \ldots \ldots \ldots$ & 13.6 & $2.1,47$ & 150,3500 & $0.34,0.65$ & 387 \\
\hline NGC $4593 \ldots \ldots \ldots$ & 5.2 & 48 & 9200 & 0.67 & 233 \\
\hline NGC $4725 \ldots \ldots \ldots$ & 16.6 & $5.1,134$ & 310,8100 & $0.37,0.58$ & 643 \\
\hline NGC $4785 \ldots . . . .$. & 4.1 & $\ldots$ & $\ldots$ & $\ldots$ & 144 \\
\hline NGC 4939 ........ & 4.7 & $\ldots$ & $\ldots$ & $\ldots$ & 337 \\
\hline NGC 4941 ........ & 32.2 & 3.5 & 110 & 0.30 & 218 \\
\hline NGC $4968 \ldots . . . .$. & 5.2 & $\ldots$ & $\ldots$ & $\ldots$ & 109 \\
\hline NGC $5033 \ldots . . . .$. & 11.0 & $0.5,3.7,30$ & $40,340,2700$ & $0.27,0.35,0.32$ & 643 \\
\hline NGC 5135 ........ & 3.8 & 40 & 10500 & 0.65 & 165 \\
\hline NGC 5194 ......... & 26.8 & $\ldots$ & $\ldots$ & $\ldots$ & 673 \\
\hline NGC $5273 \ldots \ldots \ldots$ & 9.7 & $\ldots$ & $\ldots$ & $\ldots$ & 161 \\
\hline NGC $5283 \ldots \ldots \ldots$ & 5.0 & 1.7 & 340 & 0.22 & 64 \\
\hline NGC $5347 \ldots \ldots \ldots$ & 5.6 & 31 & 5500 & 0.55 & 102 \\
\hline NGC 5427 ........ & 5.4 & $\ldots$ & $\ldots$ & $\ldots$ & 173 \\
\hline NGC $5548 \ldots \ldots \ldots$ & 3.0 & $\ldots$ & $\ldots$ & $\ldots$ & 87 \\
\hline NGC $5643 \ldots . . . .$. & 12.2 & 52 & 4300 & 0.58 & 308 \\
\hline NGC $5695 \ldots . . . .$. & 3.7 & 11 & 3000 & 0.41 & 93 \\
\hline NGC 5929 ......... & 5.4 & 1.7 & 310 & 0.23 & 59 \\
\hline NGC $5953 \ldots \ldots . .$. & 6.3 & 34 & 5400 & 0.45 & 100 \\
\hline NGC $6221 \ldots \ldots \ldots$ & 10.6 & 54 & 5100 & 0.69 & 256 \\
\hline NGC $6300 \ldots \ldots \ldots$ & 14.4 & $1.2,50$ & 80,3500 & $0.32,0.62$ & 294 \\
\hline NGC $6814 \ldots \ldots \ldots$ & 9.0 & 19 & 2100 & 0.32 & 208 \\
\hline NGC $6890 \ldots . . . .$. & 6.5 & 7.1 & 1100 & 0.38 & 93 \\
\hline NGC $6951 \ldots \ldots \ldots$ & 8.6 & 53 & 6200 & 0.64 & 287 \\
\hline NGC $7130 \ldots \ldots \ldots$ & 3.2 & 6.2 & 1900 & 0.53 & 91 \\
\hline NGC $7469 \ldots \ldots \ldots$ & 3.2 & 2.2 & 690 & 0.52 & 91 \\
\hline NGC $7479 \ldots \ldots .$. & 6.4 & 48 & 7500 & 0.74 & 256 \\
\hline NGC $7496 \ldots \ldots \ldots$ & 10.3 & 38 & 3700 & 0.79 & 199 \\
\hline NGC $7682 \ldots \ldots \ldots$ & 3.0 & 17 & 5700 & 0.54 & 77 \\
\hline NGC $7743 \ldots \ldots \ldots$ & 8.4 & 39 & 4600 & 0.48 & 181 \\
\hline UGC $1395 \ldots \ldots \ldots$ & 3.0 & 17 & 5700 & 0.46 & 77 \\
\hline
\end{tabular}

Note.-Col. (1): Galaxy names. Col. (2): Arcseconds in the galaxy image corresponding to $1 \mathrm{kpc}$. Col. (3): Deprojected bar radii in arcsec. Col. (4): Deprojected bar radii in pc. Col. (5): Deprojected bar ellipticities. Col. (6): Galaxy diameter at the 25 mag arcsec ${ }^{-2} B$ magnitude level, in arcsec, corrected for the inclination, redshift, and Galactic absorption, from RC3. Table 6 is also available in machine-readable form in the electronic edition of the Astrophysical Journal. 
TABLE 7

Bar Parameters of Non-Seyfert Control Galaxy SAmple

\begin{tabular}{|c|c|c|c|c|c|}
\hline $\begin{array}{l}\text { Galaxy } \\
\text { (1) }\end{array}$ & $\begin{array}{c}1 \mathrm{kpc} \\
(\operatorname{arcsec}) \\
(2)\end{array}$ & $\begin{array}{l}\text { Deprojected Bar Radii } \\
\text { (arcsec) } \\
(3)\end{array}$ & $\begin{array}{l}\text { Deprojected Bar Radii } \\
\text { (pc) } \\
(4)\end{array}$ & $\begin{array}{l}\text { Deprojected Bar Ellipticities } \\
\text { (5) }\end{array}$ & $\begin{array}{c}\text { Galaxy Diameter } \\
\text { (arcsec) } \\
(6)\end{array}$ \\
\hline IC $5267 \ldots \ldots \ldots$ & 9.8 & $\ldots$ & $\ldots$ & $\ldots$ & 301 \\
\hline NGC $214 \ldots \ldots .$. & 3.4 & $\ldots$ & $\ldots$ & $\ldots$ & 114 \\
\hline NGC $289 \ldots \ldots$ & 10.6 & $19^{\cdots}$ & 1800 & 0.42 & 315 \\
\hline NGC 357 ....... & 6.4 & 27 & 4200 & 0.60 & 151 \\
\hline NGC $404 \ldots . . .$. & 85.9 & $\ldots$ & $\ldots$ & $\ldots$ & 223 \\
\hline NGC $488 \ldots \ldots .$. & 7.0 & $\ldots$ & $\ldots$ & $\ldots$ & 322 \\
\hline NGC $628 \ldots . . .$. & 21.3 & 2.1 & 100 & 0.30 & 643 \\
\hline NGC $772 \ldots . . .$. & 6.3 & $\ldots$ & $\ldots$ & $\ldots$ & 455 \\
\hline NGC $864 \ldots . . .$. & 10.3 & $\ldots$ & $\ldots$ & $\ldots$ & 287 \\
\hline NGC $1345 \ldots . .$. & 11.4 & 1.4 & 120 & 0.73 & 93 \\
\hline NGC $1398 \ldots . .$. & 12.8 & 44 & 3400 & 0.52 & 425 \\
\hline NGC $1530 \ldots . .$. & 5.6 & $0.8,92$ & 150,16400 & $0.61,0.86$ & 315 \\
\hline NGC $1638 \ldots . .$. & 4.7 & $\ldots$ & $\ldots$ & $\ldots$ & 120 \\
\hline NGC $1961 \ldots \ldots$ & 3.9 & $\ldots$ & $\ldots$ & $\ldots$ & 301 \\
\hline NGC $2179 . . . .$. & 6.0 & $\ldots$ & $\cdots$ & $\cdots$ & 107 \\
\hline NGC $2196 \ldots . .$. & 7.2 & $\ldots$ & $\ldots$ & $\ldots$ & 185 \\
\hline NGC $2223 . . . .$. & 6.1 & 25 & 4100 & 0.47 & 208 \\
\hline NGC $2276 \ldots . .$. & 5.6 & 2.4 & 430 & 0.43 & 177 \\
\hline NGC $2339 . . . .$. & 6.7 & $0.8,25$ & 90,3700 & $0.53,0.59$ & 185 \\
\hline NGC $2344 \ldots . .$. & 12.9 & $\ldots$ & $\ldots$ & $\ldots$ & 112 \\
\hline NGC $2460 \ldots . .$. & 8.7 & 4.6 & 530 & 0.29 & 154 \\
\hline NGC $2566 \ldots . .$. & 9.8 & 66 & 6700 & 0.67 & 281 \\
\hline NGC $3032 \ldots . .$. & 8.4 & $\ldots$ & $\ldots$ & $\ldots$ & 117 \\
\hline NGC $3169 . . . .$. & 10.5 & 1.6 & 150 & 0.30 & 262 \\
\hline NGC $3277 . . . . .$. & 8.3 & $\ldots$ & $\ldots$ & $\ldots$ & 117 \\
\hline NGC $3300 \ldots . .$. & 4.8 & 14 & 2900 & 0.55 & 107 \\
\hline NGC $3368 \ldots . .$. & 25.5 & $4.8,77$ & 190,3000 & $0.50,0.54$ & 455 \\
\hline NGC $3865 \ldots . .$. & 2.7 & $\ldots$ & $\ldots$ & $\ldots$ & 125 \\
\hline NGC $3928 \ldots . .$. & 12.1 & $\ldots$ & $\ldots$ & $\ldots$ & 91 \\
\hline NGC $4030 \ldots . .$. & 8.0 & $\ldots$ & $\ldots$ & $\ldots$ & 256 \\
\hline NGC $4143 \ldots . .$. & 12.1 & $\ldots$ & $\ldots$ & $\ldots$ & 128 \\
\hline NGC $4254 \ldots . .$. & 12.3 & $\ldots$ & $\ldots$ & $\ldots$ & 337 \\
\hline NGC $4260 \ldots . .$. & 5.9 & 35 & 5900 & 0.49 & 161 \\
\hline NGC $4384 \ldots \ldots$ & 5.6 & 1.9 & 340 & 0.81 & 77 \\
\hline NGC $4569 . . . .$. & 12.3 & 14 & 1100 & 0.37 & 586 \\
\hline NGC $4750 \ldots . .$. & 7.9 & $2.5,14$ & 320,1800 & $0.36,0.31$ & 123 \\
\hline NGC $5054 \ldots . .$. & 7.6 & $\ldots$ & $\ldots$ & $\ldots$ & 315 \\
\hline NGC $5064 \ldots . .$. & 5.2 & $\ldots$ & $\ldots$ & $\ldots$ & 173 \\
\hline NGC $5326 \ldots . . .$. & 5.5 & $\ldots$ & $\ldots$ & $\ldots$ & 131 \\
\hline NGC $5377 \ldots . .$. & 6.7 & 74 & 11000 & 0.61 & 223 \\
\hline NGC $5383 \ldots . .$. & 5.5 & $4.4,58$ & 800,10500 & $0.43,0.63$ & 190 \\
\hline NGC $5448 \ldots . .$. & 6.3 & 49 & 7800 & 0.58 & 239 \\
\hline NGC $5614 \ldots . .$. & 4.0 & $\ldots$ & $\ldots$ & $\ldots$ & 147 \\
\hline NGC $5678 \ldots . .$. & 5.8 & 25 & 4300 & 0.46 & 199 \\
\hline NGC $5739 . . . .$. & 2.9 & $\ldots$ & $\ldots$ & $\ldots$ & 134 \\
\hline NGC $5970 \ldots . .$. & 6.5 & 12 & 1800 & 0.44 & 177 \\
\hline NGC $5985 \ldots . .$. & 5.3 & $\ldots$ & $\ldots$ & $\ldots$ & 330 \\
\hline NGC $6217 \ldots . .$. & 8.6 & 39 & 4500 & 0.72 & 185 \\
\hline NGC $6340 \ldots . .$. & 9.4 & $\ldots$ & $\ldots$ & $\ldots$ & 203 \\
\hline NGC $7096 \ldots . .$. & 5.6 & $\ldots$ & $\ldots$ & $\ldots$ & 114 \\
\hline NGC $7217 \ldots . .$. & 12.9 & $\ldots$ & $\ldots$ & $\ldots$ & 256 \\
\hline NGC $7280 \ldots . .$. & 7.9 & 1.3 & 160 & 0.27 & 128 \\
\hline NGC $7392 \ldots . .$. & 4.8 & $\ldots$ & $\ldots$ & $\ldots$ & 131 \\
\hline NGC $7421 \ldots . .$. & 8.5 & 21 & 2500 & 0.60 & 125 \\
\hline NGC $7716 \ldots . .$. & 6.1 & $3.1,23$ & 510,3800 & $0.33,0.31$ & 131 \\
\hline NGC $7742 . . . .$. & 9.3 & $1.2,6.8$ & 130,730 & $0.16,0.22$ & 107 \\
\hline
\end{tabular}

Note.-Col. (1): Galaxy names. Col. (2): Arcseconds in the galaxy image corresponding to $1 \mathrm{kpc}$. Col. (3): Deprojected bar radii in arcsec. Col. (4): Deprojected bar radii in pc. Col. (5): Deprojected bar ellipticities. Col. (6): Galaxy diameter at the 25 mag arcsec ${ }^{-2} B$ magnitude level, in arcsec, corrected for the inclination, redshift, and absorption, from RC3. Table 7 is also available in machine-readable form in the electronic edition of the Astrophysical Journal. 
Adams, T. F. 1977, ApJS, 33, 19

Antonucci, R. 1993, ARA\&A, 31, 473

Athanassoula, E. 1992, MNRAS, 259, 345

Athanassoula, E., \& Martinet, L. 1980, A\&A, 87, L10

Balick, B., \& Heckman, T. M. 1982, ARA\&A, 20, 431

Buta, R., \& Combes, F. 1996, Fundam. Cosmic Phys., 17, 95

Buta, R., \& Crocker, D. 1991, AJ, 102, 1715 .1993, AJ, 105, 1344

Colina, L., \& Wada, K. 2000, ApJ, 529, 845

Combes, F., \& Gerin, M. 1985, A\&A, 150, 327

Dahari, O. 1984, AJ, 89, 966

de Robertis, M. M., Yee, H. K. C., \& Hayhoe, K. 1998, ApJ, 496, 93

de Vaucouleurs, G. 1974, in IAU Symp. 58, The Formation and Dynamics of Galaxies, ed. J. R. Shakeshaft (Dordrecht: Reidel), 335

de Vaucouleurs, G., de Vaucouleurs, A., Corwin, H. G., Jr., Buta, R. J., Paturel, G., \& Fouque, P. 1991, Third Reference Catalogue of Bright Galaxies (New York: Springer)

Devereux, N. A., Kenney, J. D. P., \& Young, J. S. 1992, AJ, 103, 784

Elmegreen, D. M., Chromey, F. R., \& Santos, M. 1998, AJ, 116, 1221

Elmegreen, D. M., Elmegreen, B. G., \& Bellin, A. D. 1990, ApJ, 364, 415

Emsellem, E., \& Ferruit, R. 2000, A\&A, 357, 11

Erwin, P., \& Sparke, L. 1999a, ApJ, 521, L37 1999b, in ASP Conf. Ser. 182, Galaxy Dynamics, ed. D. Merritt,

J. A. Sellwood, \& M. Valluri (San Francisco: ASP), 243

Eskridge, P. B., et al. 2000, AJ, 119, 536

Forbes, D. A., Kotilainen, J. K., \& Moorwood, A. F. M. 1994, ApJ, 433, L13

Frei, Z. 1999, Ap\&SS, 269, 649

Friedli, D., Wozniak, H., Rieke, M., Martinet, L., \& Bratschi, P. 1996, A\&AS, 118, 461

Fuentes-Williams, T., \& Stocke, J. T. 1988, AJ, 96, 1235

Giovanelli, R., Haynes, M. P., Salzer, J. J., Wegner, G., da Costa, L. N., \& Freudling, W. 1994, AJ, 107, 2036

González Delgado, R. M., Heckman, T., \& Leitherer, C. 2001, ApJ, 546, 845

Goodrich, R. W., \& Osterbrock, D. E. 1983, ApJ, 269, 416

Greusard, D., Friedli, D., Wozniak, H., Martinet, L., \& Martin, P. 2000, A\&AS, 145,425

Grosbøl, P. 2001, Talk at the INAOE Workshop on Disk Galaxies, Puebla, Mexico

Ho, L. C., Filippenko, A. V., \& Sargent, W. L. W. 1997a, ApJ, 487, 591

. 1997b, ApJS, 112, 315

Huchra, J., \& Burg, R. 1992, ApJ, 393, 90

Huchra, J., Wyatt, W. F., \& Davis, M. 1982, AJ, 87, 1628

Ishizuki, S., Kawabe, R., Ishiguro, M. Okumura, S. K., \& Morita, K.-I. 1990, Nature, 344, 224

Jogee, S., Kenney, J. D. P., \& Smith, B. J. 1998, ApJ, 494, L185 .1999, ApJ, 526, 665

Jørgensen, I., Franx, M., \& Kjaergaard, P. 1992, A\&AS, 95, 489

Jungwiert, B., Combes, F., \& Axon, D. J. 1997, A\&AS, 125, 479

Knapen, J. H., Beckman, J. E., Heller, C. H., Shlosman, I., \& de Jong, R. S. 1995a, ApJ, 454, 623

Knapen, J. H., Beckman, J. E., Shlosman, I., Peletier, R. F., Heller, C. H., \& de Jong, R. S. 1995b, ApJ, 443, L73

Knapen, J. H., Shlosman, I., \& Peletier, R. F. 2000, ApJ, 529, 93 (KSP00)

Kormendy, J. 1982, ApJ, 257, 75
Kotilainen, J. K., Reunanen, J., Laine, S., \& Ryder, S. D. 2000, A\&A, 353, 834

Maciejewski, W., \& Sparke, L. 2000, MNRAS, 313, 745

MacKenty, J. W. 1989, ApJ, 343, 125

Maiolino, R., Alonso-Herrero, A., Anders, S., Quillen, A., Rieke, M. J., Rieke, G. H., \& Tacconi-Garman, L. E. 2000, ApJ, 531, 219

Márquez, I., et al. 1999, A\&AS, 140, 1

. 2000, A\&A, 360, 431

Martin, P. 1995, AJ, 109, 2428

Martinet, L., \& Friedli, D. 1997, A\&A, 323, 363

Martini, P., \& Pogge, R. W. 1999, AJ, 118, 2646

Martini, P., Pogge, R. W., Ravindranath, S., \& An, J. H. 2001, ApJ, 562, 139

Mirabel, I. F., et al. 1999, A\&A, 341, 667

Moles, M., Márquez, I., \& Pérez, E. 1995, ApJ, 438, 604

Möllenhoff, C., Matthias, M., \& Gerhard, O. E. 1995, A\&A, 301, 359

Mulchaey, J. S., \& Regan, M. W. 1997, ApJ, 482, L135

Noguchi, M. 1988, A\&A, 203, 259

Peletier, R. F., Balcells, M., Davies, R. L., Andredakis, Y., Vazdekis, A., Burkert, A., \& Prada, F. 1999a, MNRAS, 310, 703

Peletier, R. F., Knapen, J. H., Shlosman, I., Pérez-Ramírez, D., Nadeau, D., Doyon, R., Rodriguez Espinosa, J. M., \& Pérez García, A. M. 1999b, ApJS, 125, 363

Peletier, R. F., Valentijn, E. A., Moorwood, A. F. M., Freudling, W., Knapen, J. H., \& Beckman, J. E. 1995, A\&A, 300, L1

Pfenniger, D., \& Norman, C. 1990, ApJ, 363, 391

Quillen, A. C., Alonso-Herrero, A., Rieke, M. J., Rieke, G. H., Ruiz, M., \& Kulkarni, V. 1999, ApJ, 527, 696

Quillen, A. C., McDonald, C., Alonso-Herrero, A., Lee, A., Shaked, S., Rieke, M. J., \& Rieke, G. H. 2001, ApJ, 547, 129

Regan, M. W., \& Mulchaey, J. S. 1999, AJ, 117, 2676

Rieke, G. H., \& Lebofsky, M. J. 1985, ApJ, 288, 618

Salo, H. 1991, A\&A, 243, 118

Sandage, A., \& Brucato, R. 1979, AJ, 84, 472

Schmitt, H. R. 2001, AJ, 122, 2243

Schwarz, M. P. 1984, MNRAS, 209, 93

Scoville, N. Z., et al. 2000, AJ, 119, 991

Seigar, M. Carollo, M., Dejonghe, H., Stiavelli, M., \& de Zeeuw, T. 2000, in ASP Conf. Ser. 197, Dynamics of Galaxies: From the Early Universe to the Present, ed. G. A. Mamon \& V. Charmandaris (San Francisco: ASP), 269

Shaw, M. A., Axon, D. J., Probst, R., \& Gatley, I. 1995, MNRAS, 274, 369

Shaw, M. A., Combes, F., Axon, D. J., \& Wright, G. S. 1993, A\&A, 273, 31

Sheth, K., Regan, M. W., Vogel, S. N., \& Teuben, P. J. 2000, ApJ, 532, 221

Shlosman, I., Frank, J., \& Begelman, M. C. 1989, Nature, 338, 45

Shlosman, I., \& Heller, C. H. 2002, ApJ, 565, 921

Shlosman, I., Peletier, R. F., \& Knapen, J. H. 2000, ApJ, 535, L83

Simkin, S. M., Su, H. J., \& Schwarz, M. P. 1980, ApJ, 237, 404

Thompson, R. I., \& Corbin, M. 1999, Ap\&SS, 266, 79

Tully, R. B. 1988, Nearby Galaxies Catalog (Cambridge: Cambridge Univ. Press)

van den Bosch, F. C., \& Emsellem, E. 1998, MNRAS, 298, 267

Veron, M. P., Veron, P., \& Zuiderwijk, E. J. 1981, A\&A, 98, 34

Veron-Cetty, M.-P., \& Veron, P. 1991, A Catalogue of Quasars and Active Nuclei (5th ed.; Garching: ESO)

Wozniak, H., Friedli, D., Martinet, L., Martin, P., \& Bratschi, P. 1995, A\&AS, 111, 115 